\title{
Practical applications of averages and differences of Friedel opposites
}

\author{
H. D. Flack, M. Sadki, A. L. Thompson and D. J. Watkin
}

Acta Cryst. (2011). A67, 21-34

Copyright (C) International Union of Crystallography

Author(s) of this paper may load this reprint on their own web site or institutional repository provided that this cover page is retained. Republication of this article or its storage in electronic databases other than as specified above is not permitted without prior permission in writing from the IUCr.

For further information see http://journals.iucr.org/services/authorrights.html

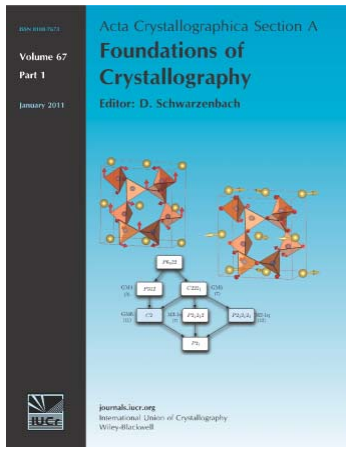

Acta Crystallographica Section A: Foundations of Crystallography covers theoretical and fundamental aspects of the structure of matter. The journal is the prime forum for research in diffraction physics and the theory of crystallographic structure determination by diffraction methods using X-rays, neutrons and electrons. The structures include periodic and aperiodic crystals, and non-periodic disordered materials, and the corresponding Bragg, satellite and diffuse scattering, thermal motion and symmetry aspects. Spatial resolutions range from the subatomic domain in charge-density studies to nanodimensional imperfections such as dislocations and twin walls. The chemistry encompasses metals, alloys, and inorganic, organic and biological materials. Structure prediction and properties such as the theory of phase transformations are also covered.

\section{Crystallography Journals Online is available from journals.iucr.org}


Acta Crystallographica Section A

Foundations of Crystallography

ISSN 0108-7673

Received 14 September 2010

Accepted 21 October 2010

\section{Practical applications of averages and differences of Friedel opposites}

\author{
H. D. Flack, ${ }^{a}$ M. Sadki, ${ }^{b}$ A. L. Thompson ${ }^{b}$ and D. J. Watkin ${ }^{\text {b* }}$ \\ a'Département de Chimie Minérale, Analytique et Appliquée, University of Geneva, Switzerland, \\ and ${ }^{\mathbf{b}}$ Chemical Crystallography, Chemistry Research Laboratory, Department of Chemistry, \\ University of Oxford, OX1 3TA, England. Correspondence e-mail: david.watkin@chem.ox.ac.uk
}

(C) 2011 International Union of Crystallography Printed in Singapore - all rights reserved
The practical use of the average and difference intensities of Friedel opposites at different stages of structure analysis has been investigated. It is shown how these values may be properly and practically used at the stage of space-group determination. At the stage of least-squares refinement, it is shown that increasing the weight of the difference intensities does not improve their fit to the model. The correct form of the coefficients for a difference electron-density calculation is given. In the process of structure validation, it is further shown that plots of the observed and model difference intensities provide an objective method to evaluate the fit of the data to the model and to reveal insufficiencies in the intensity measurements. As a further tool for the validation of structure determinations, the use of the Patterson functions of the average and difference intensities has been investigated and their clear advantage demonstrated.

\section{Introduction}

It is the main contention of this paper that the differences in intensity between Friedel opposites $h k l$ and $\overline{h k l}$ are at present insufficiently exploited in small-molecule single-crystal structure analysis. This neglect shows itself at various stages of structure analysis. Here we concern ourselves with spacegroup determination, least-squares refinement, electrondensity calculation and structure validation. Current practice is to regard Friedel differences only of interest in the determination of absolute structure, but by selected examples we show that there is a far wider domain of applicability.

The simple technique applied is that, rather than working directly with the observed and model values of $|\mathbf{F}(h k l)|^{2}$ and $|\mathbf{F}(\overline{h k l})|^{2}$, we prefer to use their average $(A)$ and difference (D) values given by

$$
\begin{gathered}
A(h k l)=\frac{1}{2}\left[|F(h k l)|^{2}+|F(\overline{h k l})|^{2}\right], \\
D(h k l)=|F(h k l)|^{2}-|F(\overline{h k l})|^{2} .
\end{gathered}
$$

This leads naturally to the Bijvoet ratio defined by

$$
\chi=\left\langle D^{2}\right\rangle^{1 / 2} /\langle A\rangle
$$

as the ratio of the root-mean-square value of $D$ to the mean value of $A$. In a structure analysis, two independent estimates of the Bijvoet ratio are available. The first arises from considerations of intensity statistics through the analysis of an ensemble of random structures (Flack \& Shmueli, 2007; Shmueli et al., 2008; Shmueli \& Flack, 2009) leading to the definition of the Bijvoet ratio as a value called Friedif stat $_{\text {, }}$,

$$
\text { Friedif }_{\text {stat }}=2 \times 10^{4} \frac{\left\{\sum_{i=1}^{N} \sum_{j=1}^{N}\left[\left(f_{i}^{\mathrm{o}}+f_{i}^{\prime}\right) f_{j}^{\prime \prime}-f_{i}^{\prime \prime}\left(f_{j}^{\mathrm{o}}+f_{j}^{\prime}\right)\right]^{2}\right\}^{1 / 2}}{\sum_{j=1}^{N}\left[\left(f_{j}^{\mathrm{o}}+f_{j}^{\prime}\right)^{2}+f_{j}^{\prime 2}\right]} .
$$

In equation (4) the numerator and denominator are proportional, respectively, to the root-mean-square value of $D$ and the mean value of $A$. One needs only to know the chemical composition of the compound and the wavelength of the $\mathrm{X}$-radiation used to evaluate equation (4). Friedif stat $_{\text {has man }}$ interesting properties, not the least being that a pair of different atoms of the same chemical element make a contribution of zero to the numerator. Friedif stat $_{\text {takes values around }}$ 1000 for a compound with atoms some of which are strong resonant scatterers, whereas for a compound containing only light chemical elements $(Z<10)$ Friedif $_{\text {stat }}$ falls well below 100. The second estimate of the Bijvoet ratio, Friedif ${ }_{\text {obs }}$, is obtained from the observed diffraction intensities. Flack \& Bernardinelli (2008) (hereafter FB2008) made an initial study of Friedif $_{\text {obs }}$ in practical applications and this is more fully developed in $\S 3$ of the current paper.

The use of $A(h k l)$ and $D(h k l)$ instead of $|\mathbf{F}(h k l)|^{2}$ and $|\mathbf{F}(\overline{h k l})|^{2}$ imposes certain restrictions on the intensity data. For acentric reflections one needs to have measured both $h k l$ and $\overline{h k l}$, whereas for centric reflections only one of the pair is necessary. In order to assign a reflection as being acentric or centric, and to identify its symmetry-equivalent reflections, the crystal point group has to be known. Now the latter is usually only definitely determined during the process of crystal structure determination. Consequently the best approach is to 
measure a whole sphere of reflections out to a chosen resolution limit. The next most satisfactory way is to measure a complete asymmetric unit of reflections $h k l$ of the Laue class (identified from the lattice metric and point-group symmetry of reciprocal space) together with their exact Friedel opposites $\overline{h k l}$. In relation to reflections, the terms acentric and centric are defined in Shmueli \& Flack (2009) and tables are provided therein to classify reflections according to point group.

Concerning the order of the sections in this paper, we have chosen the way in which they would arise in structure analysis. The only exception is $\S 2$ on the comparison of $D_{\text {obs }}$ and $D_{\text {model }}$, properly part of structure validation, which, because of its importance in the other sections of the work, is presented directly after this introduction. In this paper, equations are written in an expanded form with respect to our previous work in an attempt to make them accessible to a wider audience. Moreover, contrary to our previous approach, the real part of the resonant scattering contribution is included explicitly.

\section{Comparing $D_{\text {obs }}$ and $D_{\text {model }}$}

We have re-examined the structure determinations presented in $\S 3$ and Tables 3, 4 and 5 of FB2008. It turned out that a fruitful way of presenting and analysing the data had escaped our attention at that time. The method that we now demonstrate consists of plotting $D_{\text {obs }}$ versus $D_{\text {model }}$. For the reader's information, we recall that the non-centrosymmetric structure determinations used by FB2008 were mainly published in Acta Crystallographica B, C and E in the first months of 2007. Consequently files containing the intensity data were available. In the course of this work we have studied these structure analyses with a very critical eye and our comments are collected together in the supplementary material accompanying this paper. ${ }^{\mathbf{1}}$ None of these structures are mentioned in Marsh (2009) as having an incorrect space-group assignment nor have they been retracted from publication (Harrison et al., 2010; IUCr Editorial Office, 2010).

We arranged these structure determinations in order of increasing $R_{D}$, a conventional $R$ factor on the values of $D$,

$$
R_{D}=\sum_{h k l}^{\text {paired acentric }}\left|D_{\text {obs }}(h k l)-D_{\text {model }}(h k l)\right| / \sum_{h k l}^{\text {paired acentric }}\left|D_{\text {obs }}(h k l)\right|
$$

(see Table 5 and Glossary of FB2008). Compounds are identified by their CSD refcodes (see Table 1). Values of $R_{D}$ range from $40.2 \%$ for EZEQAB to $100 \%$ for SEZPUJ. For each structure determination, the plots were prepared so that the range and dimensions of the $D_{\text {obs }}$ and $D_{\text {model }}$ axes were always identical. In some plots the data were limited to specified ranges of $A_{\text {obs. }}$ Other structure determinations of our own, not described here, confirmed the behaviour of the data sets of FB2008.

\footnotetext{
${ }^{1}$ Notes on structure determinations analysed in this paper are available from the IUCr electronic archives (Reference: SH5118). Services for accessing these data are described at the back of the journal.
}

Table 1

Structure determinations taken from FB2008.

REFCODE is either that of the CSD (Cambridge Structural Database; Allen, 2002) or a local code beginning with $9 . R_{F}$ is the unweighted $R$ factor given in the original publication of the structure as a percentage. $R_{A}=\sum_{h k l}^{\text {paired acentric }}\left|A_{\text {obs }}(h k l)-A_{\text {model }}(h k l)\right| / \sum_{h k l}^{\text {paired acentric }}\left|A_{\text {obs }}(h k l)\right|$ is an unweighted $R$ factor on the Friedel average intensities as a percentage. $R_{D}=\sum_{h k l}^{\text {paired acentric }}\left|D_{\text {obs }}(h k l)-D_{\text {model }}(h k l)\right| / \sum_{h k l}^{\text {paired acentric }}\left|D_{\text {obs }}(h k l)\right|$ is an unweighted $R$ factor on the Friedel-difference intensities as a percentage. The Flack parameter is defined in Flack (1983). Friedif are values of the

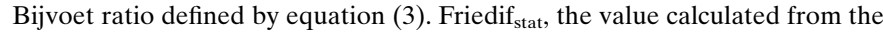
chemical composition and the wavelength, is defined by equation (4) at $\sin \theta / \lambda$ $=0$. Friedif ${ }_{\text {obs }}$, a value calculated from the observed intensity data, is described in full in $\$ 3$. The upper part of the table (EZEQAB to CICYIX) contains compounds with a good fit of $D_{\text {obs }}$ to $D_{\text {model }}$ and low values of $R_{D}$. The lower part of the table (KEXYOC to YIFZAP) contains compounds with a bad fit of $D_{\text {obs }}$ to $D_{\text {model }}$ and high values of $R_{D}$. The middle part of the table (METSIO to RIGMAW) contains compounds with an intermediate fit of $D_{\text {obs }}$ to $D_{\text {model }}$.

\begin{tabular}{|c|c|c|c|c|c|c|}
\hline REFCODE & $R_{F}(\%)$ & $R_{A}(\%)$ & $R_{D}(\%)$ & $\begin{array}{l}\text { Flack } \\
\text { parameter }\end{array}$ & Friedif $_{\text {stat }}$ & Friedif $_{\text {obs }}$ \\
\hline EZEQAB & 3.6 & 1.2 & 40.2 & $-0.03(11)$ & 86 & 291 \\
\hline YIDJIF & 2.5 & 4.4 & 45.8 & $0.041(5)$ & 700 & 736 \\
\hline WIGWUF & 3.2 & 5.2 & 47.8 & $-0.016(10)$ & 937 & 1374 \\
\hline GIHKEO & 3.4 & 4.3 & 52.7 & $-0.018(12)$ & 949 & 1290 \\
\hline XICNED & 1.5 & 2.8 & 52.7 & $0.012(7)$ & 421 & 779 \\
\hline TICFIV & 2.1 & 3.0 & 54.1 & $-0.01(2)$ & 370 & 866 \\
\hline CICXES & 3.2 & 4.1 & 56.0 & $0.015(8)$ & 386 & 505 \\
\hline METWIS & 2.8 & 4.1 & 65.2 & $0.010(10)$ & 570 & 267 \\
\hline GIHDAD & 2.3 & 3.7 & 70.6 & $0.0620(10)$ & 802 & 945 \\
\hline CICYIX & 1.8 & 3.2 & 71.0 & $-0.002(17)$ & 314 & 501 \\
\hline METSIO & 3.4 & 3.6 & 57.1 & $-0.021(3)$ & 1351 & 1577 \\
\hline TIBCAJ & 4.4 & 4.1 & 62.8 & $-0.039(14)$ & 1258 & 2288 \\
\hline 9BER01 & 2.8 & 3.2 & 66.2 & $0.00(3)$ & 172 & 299 \\
\hline RIHMUR & 1.7 & 2.0 & 72.5 & $-0.02(3)$ & 198 & 320 \\
\hline CIKCUV & 2.3 & 3.7 & 78.2 & $-0.05(4)$ & 119 & 228 \\
\hline PIFDOY & 3.1 & 4.9 & 81.5 & $0.011(19)$ & 531 & 623 \\
\hline PEFXII & 2.5 & 1.8 & 84.8 & $-0.01(2)$ & 865 & 1067 \\
\hline 9YAN01 & 4.5 & 6.8 & 87.0 & $-0.01(3)$ & 616 & 1299 \\
\hline RIGHEV & 6.4 & 8.5 & 94.6 & $0.05(3)$ & 253 & 989 \\
\hline RIGMAW & 4.6 & 7.1 & 98.2 & $-0.02(6)$ & 89 & 1493 \\
\hline KEXYOC & 3.4 & 4.8 & 92.2 & $-0.05(5)$ & 110 & 522 \\
\hline XIFSIP & 3.3 & 4.3 & 94.0 & $0.00(5)$ & 104 & 382 \\
\hline UDUSIW & 3.4 & 4.7 & 96.6 & $-0.03(7)$ & 74 & 377 \\
\hline SIHDET & 4.7 & 7.6 & 97.1 & $-0.02(8)$ & 75 & 590 \\
\hline EDUZOT & 2.2 & 3.1 & 97.2 & $0.04(6)$ & 108 & 501 \\
\hline UNEVAK01 & 4.1 & 4.9 & 97.7 & $0.02(10)$ & 70 & 499 \\
\hline CIJWUO & 7.4 & 5.5 & 98.3 & $0.41(3)$ & $784 / 141$ & 1800 \\
\hline SEZPUJ & 4.3 & 6.3 & 100 & $0.12(11)$ & 81 & 2930 \\
\hline YIFZAP & 7.4 & 8.2 & 101 & $-0.1(3)$ & 100 & 1564 \\
\hline
\end{tabular}

Fig. 1 shows plots of $D_{\text {obs }}$ versus $D_{\text {model }}$ for EZEQAB which has the lowest $R_{D}$ value in FB2008. For Fig. 1(a) all data are presented, whereas for Fig. 1(b) only data with $A_{\text {obs }}<1 \% A_{\max }$ and for Fig. $1(c)$ only data with $A_{\text {obs }}>10 \% A_{\max }$ are presented. The value of $R_{A}$, a conventional $R$ factor on the values of $A$,

$$
R_{A}=\sum_{h k l}^{\text {paired acentric }}\left|A_{\text {obs }}(h k l)-A_{\text {model }}(h k l)\right| / \sum_{h k l}^{\text {paired acentric }}\left|A_{\text {obs }}(h k l)\right|
$$

(see Table 5 and Glossary of FB2008), is $1.2 \%$ and that of $R_{D}$ is $40.2 \%$. All three graphs show that the data points follow a straight line of slope 1 (i.e. at $45^{\circ}$ to the two axes) passing through the origin. The ranges of data values in $D_{\text {obs }}$ and $D_{\text {model }}$ are closely similar. Moreover, there is no change of 


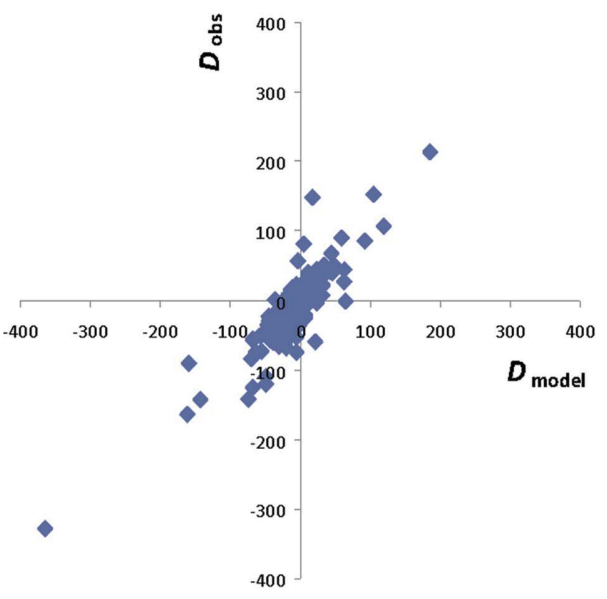

(a)

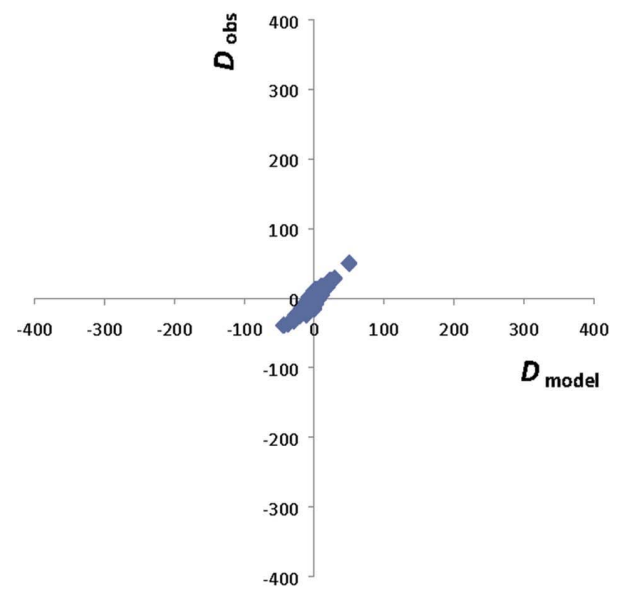

(b)

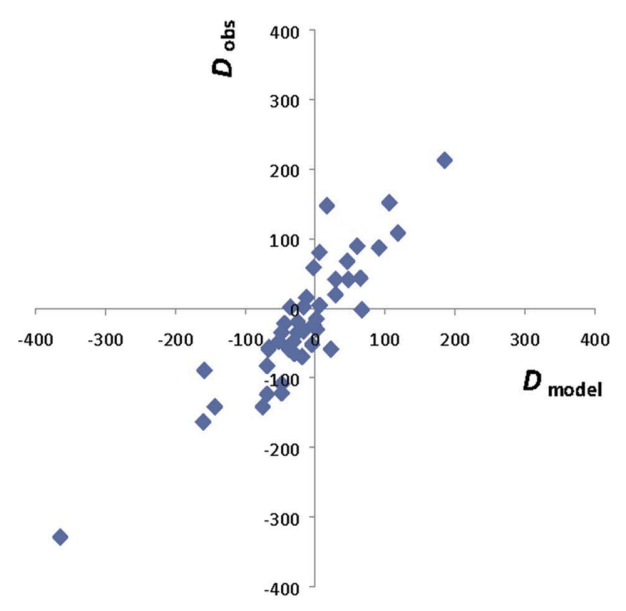

(c)

Figure 1

Plots of $D_{\text {obs }}$ versus $D_{\text {model }}$ for EZEQAB which has $R_{A}=1.2 \%$ and $R_{D}=$ 40.2\%. (a) Displays all data, (b) data with $A_{\text {obs }}<1 \% A_{\max }$ and (c) data with $A_{\text {obs }}>10 \% A_{\text {max }}$. behaviour on limiting the data to reflections with large or small values of $A_{\text {obs }}$. Indeed these data plots of EZEQAB are 'what one expects' and what one would hope to obtain in a satisfactory structure analysis. In a general way, the intensity data are good, the model provides a satisfactory description of the Friedel differences and the least-squares refinement has done a reasonable job in fitting $D_{\text {model }}$ to $D_{\text {obs }}$. Further comments and results on the least-squares refinement are presented in \$4.1. The structure determinations in FB2008 which follow the same pattern as EZEQAB are given in the upper part of Table 1. Apart from EZEQAB itself, which has a

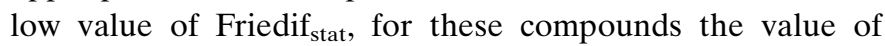
Friedif $_{\text {stat }}$ is medium or high. They also have among the lowest values of $R_{D}$.

Fig. 2 shows plots of $D_{\text {obs }}$ versus $D_{\text {model }}$ for SEZPUJ which has one of the highest $R_{D}$ values in FB2008. For Fig. 2(a) all data are presented, whereas for Fig. 2(b) only data with $A_{\text {obs }}<$ $0.1 \% A_{\max }$ and for Fig. 2(c) only data with $A_{\text {obs }}>2 \% A_{\max }$ are presented. The value of $R_{A}$ is $6.3 \%$ and that of $R_{D}$ is $100 \%$. All three graphs show data spread along the $D_{\text {obs }}$ axis with a range of $D_{\text {obs }}$ values that is much greater than that of $D_{\text {model }}$. There is no sign at all of a scatter of points around a straight line of slope 1 . This scattergram of SEZPUJ is definitely not 'what one expects' and not at all what one wants from a satisfactory structure analysis. The value of $R_{D}(100 \%)$ very well reflects the disagreement between the $D_{\text {obs }}$ and $D_{\text {model }}$ values of SEZPUJ. From the authors' conventional $R$ values and FB2008's $R_{A}$ values, this is a satisfactorily refined structure. It is unimaginable that further shifts in the atomic parameters could maintain the agreement between $A_{\text {obs }}$ and $A_{\text {model }}$ whilst increasing the values of $D_{\text {model }}$ by the factor of 10 necessary for the required scatter around a line of slope 1 to appear. Consequently one is driven to the conclusion that the problem is entirely in the intensity data themselves. These have produced a satisfactory set of $A_{\text {obs }}$ values whilst at the same time resulting in $D_{\text {obs }}$ values that are apparently valueless and lack information on the intensity differences between Friedel opposites due to resonant scattering. Other structure determinations in FB2008 which follow the same pattern as SEZPUJ are given in the lower part of Table 1. The compounds in this class tend to have low values of Friedif stat $_{\text {. }}$

The rest of the structure determinations in FB2008 display behaviours intermediate between those of EZEQAB and SEZPUJ and are presented in the middle part of Table 1. The range of values of $D_{\text {obs }}$ is larger than that of $D_{\text {model }}$ but not as pronounced as in SEZPUJ.

METSIO and TIBCAJ show the behaviour of SEZPUJ for small values of $A_{\mathrm{obs}}$ and that of EZEQAB for intermediate and large values of $A_{\mathrm{obs}}$.

9YAN01, RIGHEV and RIGMAW show the behaviour of SEZPUJ for small and intermediate values of $A_{\mathrm{obs}}$ and that of EZEQAB for large values of $A_{\text {obs }}$.

9BER01 and CIKCUV show the behaviour of EZEQAB for intermediate values of $A_{\text {obs. }}$. For small and large values of $A_{\text {obs }}$, there is a clear tendency for $D_{\text {obs }}$ to be larger than $D_{\text {model }}$.

RIHMUR and PIFDOY show the behaviour of EZEQAB for small and intermediate values of $A_{\text {obs. }}$. For large values 


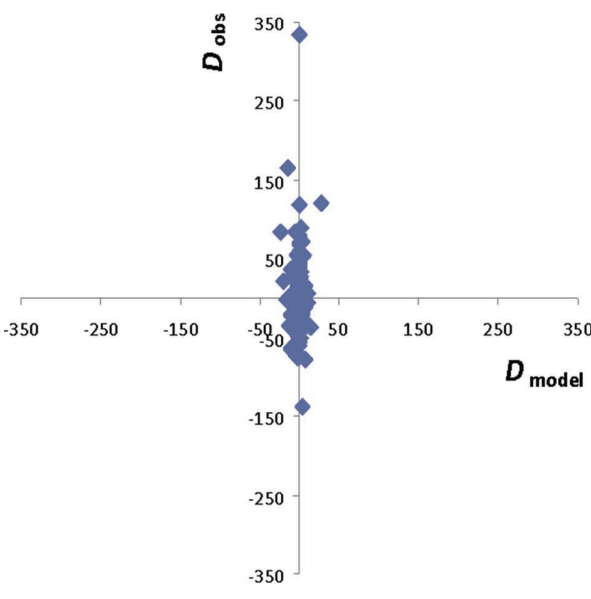

(a)

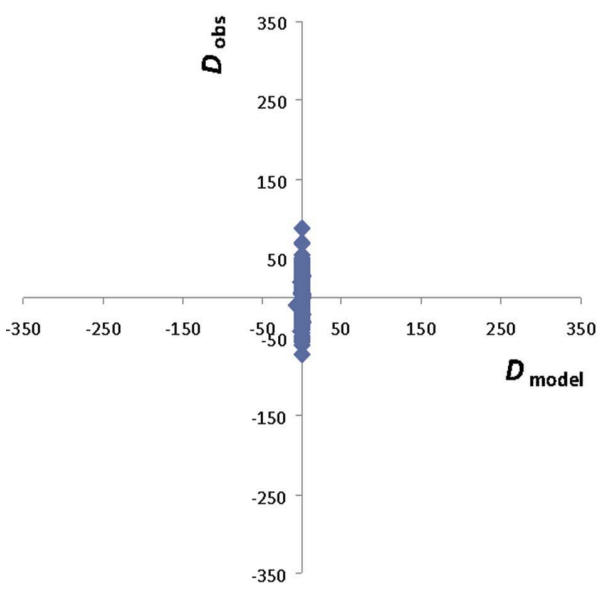

(b)

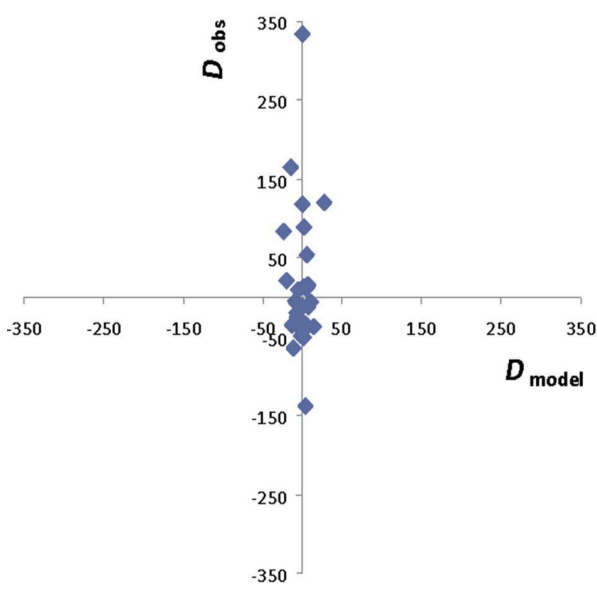

(c)

Figure 2

Plots of $D_{\text {obs }}$ versus $D_{\text {model }}$ for SEZPUJ which has $R_{A}=6.3 \%$ and $R_{D}=$ $100 \%$. (a) Displays all data, (b) data with $A_{\text {obs }}<0.1 \% A_{\max }$ and (c) data with $A_{\text {obs }}>2 \% A_{\text {max }}$. of $A_{\text {obs}}$, there is a clear tendency for $D_{\text {obs }}$ to be larger than $D_{\text {model }}$.

PEFXII shows the behaviour of EZEQAB for small values of $A_{\text {obs. }}$ For intermediate and large values of $A_{\text {obs, }}$, there is a clear tendency for $D_{\text {obs }}$ to be larger than $D_{\text {model }}$.

As an example, Fig. 3 shows the plots for CIKCUV.

As a further check, we also examined other plots of EZEQAB, TICFIV, SIHDET, SEZPUJ, METSIO and RIGMAW. For these compounds, plots of $D_{\text {obs }}$ versus $D_{\text {model }}$ were made limiting the data to low and then high values of $\sin \theta / \lambda$. These plots looked very much like those for large and small $A_{\text {obs }}$, respectively. One is hardly surprised by this result and there were no other significant features in these plots. Plots were also made of $A_{\text {obs }}$ versus $A_{\text {model }}$ on logarithmic axes. These showed the ordinary appearance of a straight line of slope 1 passing through the origin. In general there was more scatter of points at small values of $A$. EZEQAB appears to be an exceptionally good data set.

As a reference, it is of interest to have in mind the form of the $D_{\text {obs }}$ versus $D_{\text {model }}$ plot for a centrosymmetric crystal structure. In this case, all $D_{\text {model }}(h k l)=0$ although the $D_{\text {obs }}(h k l)$ will show values different from zero because of random and systematic effects. Consequently all data points will be placed at $D_{\text {model }}=0$ on the $D_{\text {obs }}$ axis. In this way, centrosymmetric structures look like an extreme case of the structures in the lower part of Table 1 and have a value of $R_{D}=$ $100 \%$. This statement should not be taken to imply that these crystal structures are in fact centrosymmetric but only that their $D_{\text {obs }}$ values are dominated by random and systematic effects unrelated to intensity differences in Friedel opposites due to resonant scattering, as is the case for any centrosymmetric structure.

\section{Space-group determination}

Current practice in the determination of the space group of a crystal structure follows that of Looijenga-Vos \& Buerger (2002). Many common software implementations follow this approach. It proceeds in two stages: $(a)$ analysis of the equivalence of diffraction intensities to determine the Laue class of the crystal, followed by $(b)$ analysis of reflections of negligible intensity to reveal the conditions for reflection (i.e. identification of space-group-absent reflections) followed by the assignment of possible space groups. The use in stage $(a)$ of the Laue class is justified by Looijenga-Vos \& Buerger (2002) by assuming that Friedel's law is valid even in the presence of resonant scatterers. This assumption is in fact unnecessary. The whole of the Looijenga-Vos \& Buerger (2002) procedure is entirely valid if one works with the $A_{\text {obs }}(h k l)$ of the Friedel opposites of acentric reflections rather than on the individual intensities $|\mathbf{F}(h k l)|^{2}$ obs. In some cases, the procedure leads to multiple choices for the space group of the crystal, some of which are centrosymmetric and some are noncentrosymmetric. Most often, structure analysts use the results of intensity statistics of the average observed intensity of Friedel opposites, $A(h k l)_{\text {obs }}$, to attempt to distinguish between a centrosymmetric and a non-centrosymmetric choice of space 


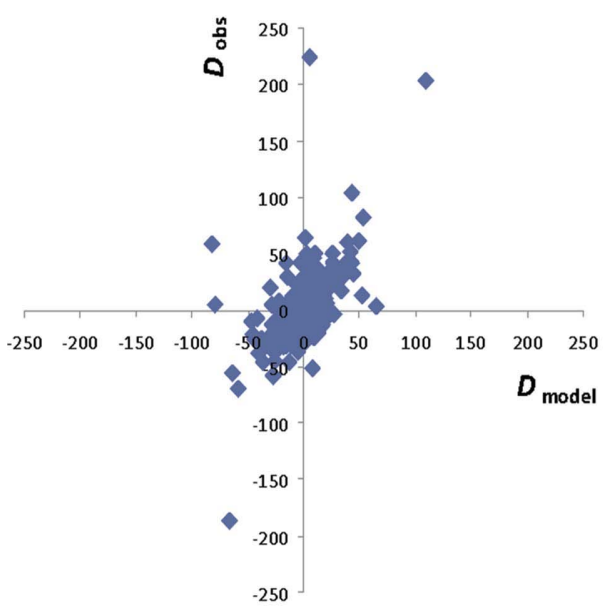

(a)

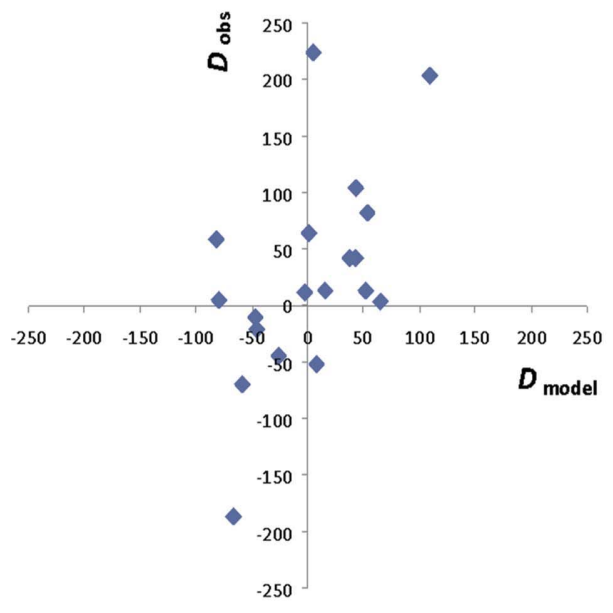

(c)

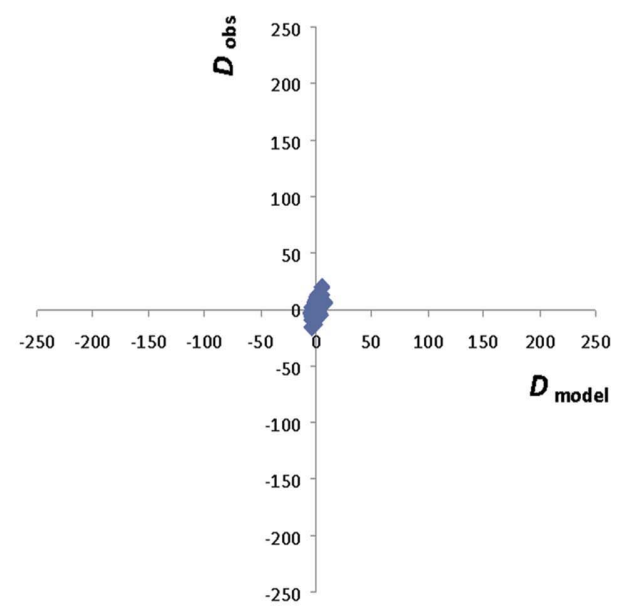

(b)

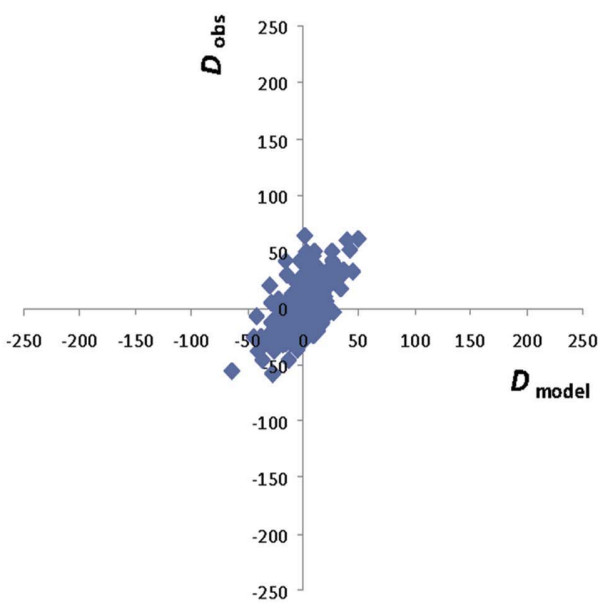

(d)

Figure 3

Plots of $D_{\text {obs }}$ versus $D_{\text {model }}$ for CIKCUV which has $R_{A}=3.7 \%$ and $R_{D}=78.2 \%$. (a) Displays all data, $(b)$ data with $A_{\text {obs }}<0.3 \% A_{\text {max }},(c)$ data with $A_{\text {obs }}>$ $15 \% A_{\max }$ and $(d)$ data with $0.3 \% A_{\max }<A_{\text {obs }}<15 \% A_{\max }$.

group. However, in this approach and that of Looijenga-Vos \& Buerger (2002) and common software implementations, no use or analysis is made of the $D(h k l)_{\text {obs }}$ to differentiate between the various choices of space group.

We proceed by calculating a value of the Bijvoet ratio [equation (3)], Friedif ${ }_{\text {obs }}$, from the observed values of $A$ and $D$, and comparing it with Friedif stat $_{\text {based on statistical }}$ considerations (see §1). Previous works (Flack \& Shmueli, 2007; Shmueli et al., 2008; Shmueli \& Flack, 2009; FB2008) have shown that $A$ and $D$ do not have the same dependence on $\sin \theta / \lambda$. It is thus essential, as far as possible, to eliminate this difference in calculating a value of Friedif ${ }_{\text {obs }}$. Consequently our spreadsheet application (Flack \& Shmueli, 2007; FB2008) was further extended to undertake the calculation of

\footnotetext{
${ }^{2}$ A spreadsheet application for the calculation of Friedif stat $_{\text {discussed in this }}$ paper is available from the IUCr electronic archives (Reference: SH5118). Services for accessing these data are described at the back of the journal.
}

Friedif $_{\text {stat }}$ at values of $\sin \theta / \lambda$ from 0.0 to $0.7 \AA^{-1}$ in steps of $0.05 \AA^{-1}$. It is available as supplementary material. ${ }^{2}$ Moreover, the calculation now includes $f^{\prime}$, the real part of the resonant scattering contribution. The values of Friedif $f_{\text {stat }}$, r.m.s. $D$ and $\langle A\rangle$ are tabulated at the above values of $\sin \theta / \lambda$ for the three radiations $\mathrm{Cr} K \alpha, \mathrm{Cu} K \alpha$ and Mo $K \alpha$. Graphs of Friedif $_{\text {stat }}$ versus $\sin \theta / \lambda$ and $\langle A\rangle$ versus $(\sin \theta / \lambda)^{2}$, and polynomial fits to these functions are also produced. The plot of Friedif $_{\text {stat }}$ versus $\sin \theta / \lambda$ is shown in Fig. 4(a) for the compound TIBCAJ. In applying these values to an intensity data set, the values of the overall scale factor and isotropic displacement parameter are found from a Wilson plot of $\log \left(A_{\text {obs }} /\langle A\rangle\right)$ versus $(\sin \theta / \lambda)^{2}$ allowing normalized $A_{\text {obs }}$ values to be obtained. A scatter diagram of the latter shows them to be essentially independent of $\sin \theta / \lambda$ (see Fig. $4 b$ ) and allows $\left\langle A_{\text {obs }}\right\rangle$ to be evaluated for the normalized values. The values of $D_{\text {obs }}$ (see Fig. $4 c$ ) are preliminarily normalized in the same way using the same $\langle A\rangle$ 's, overall scale factor and overall isotropic 


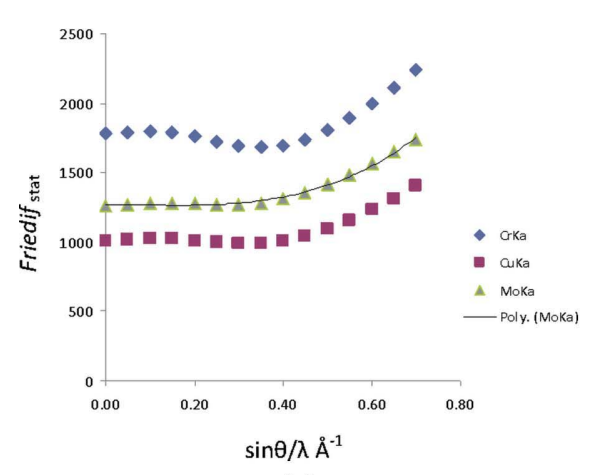

$(a)$

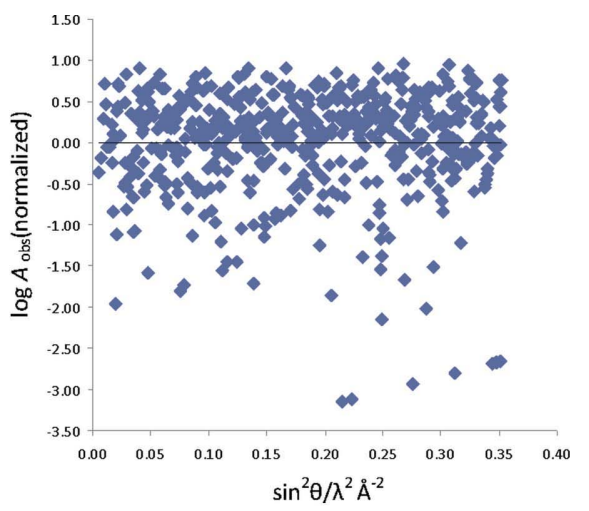

(b)

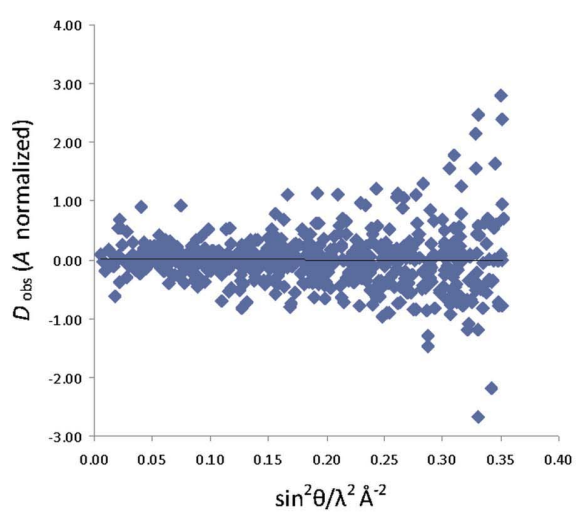

$(d)$

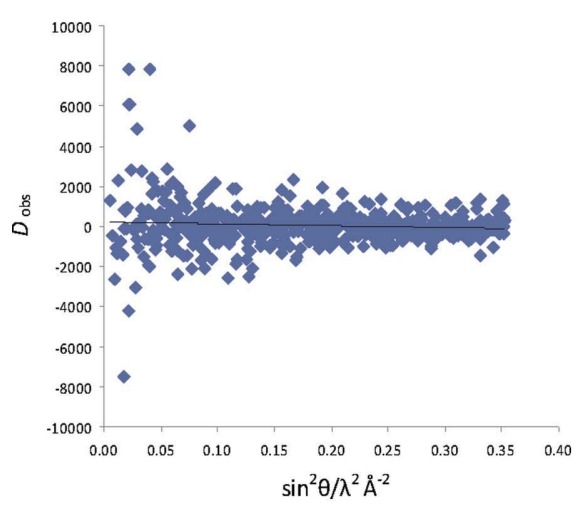

(c)

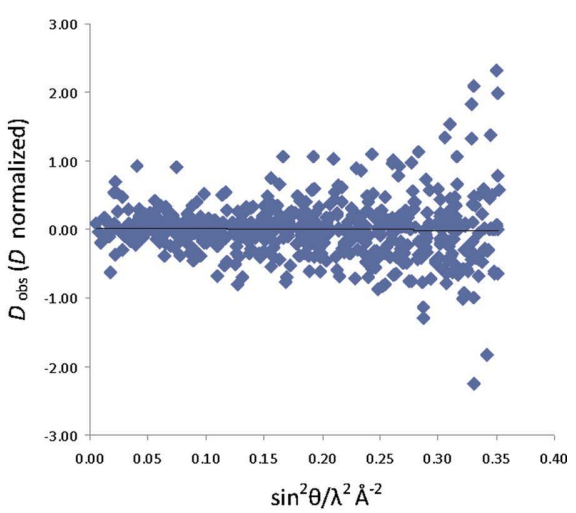

$(e)$

Figure 4

Plots for the compound TIBCAJ. ( $a$ ) The variation of Friedif stat $_{\text {with }} \sin \theta / \lambda$ for the radiations $\mathrm{Cr} K \alpha, \mathrm{Cu} K \alpha$ and Mo $K \alpha$. The polynomial representation of Friedif $_{\text {stat }}$ for Mo $K \alpha$ is Friedif stat $=2072.2(\sin \theta / \lambda)^{3}-434.19(\sin \theta / \lambda)^{2}-23.951 \sin \theta / \lambda+1269.1$. (b) A scatter plot of normalized log $A_{\text {obs }} v e r s u s$ $(\sin \theta / \lambda)^{2}$. (c) A scatter plot of $D_{\text {obs }}$ versus $(\sin \theta / \lambda)^{2}$. $(d)$ A similar scatter plot of $D_{\text {obs }}$ values normalized according to the procedure applied to the values of $A_{\text {obs. }}$ ( $e$ ) The scatter plot of $D_{\text {obs }}$ values further adjusted to allow for the variation of Friedif stat $_{\text {with }} \sin \theta / \lambda$.

atomic displacement parameter (see Fig. 4d). However, for the $D_{\text {obs }}$ a further adjustment is made to allow for the $\sin \theta / \lambda$

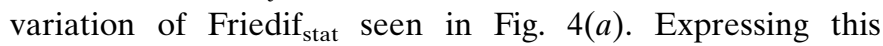
variation as a polynomial, Friedif stat $=c_{0}+c_{1} \sin \theta / \lambda+$ $c_{2}(\sin \theta / \lambda)^{2}+\ldots$, the preliminary normalized $D_{\text {obs }}$ are modified to become $D_{\text {obs }} /\left[1+c_{1} / c_{0} \sin \theta / \lambda+c_{2} / c_{0}(\sin \theta / \lambda)^{2}\right]$. Although this additional correction reduced the $\sin \theta / \lambda$ dependence of the normalized $D_{\text {obs }}$ for most of the compounds in Table 1 , some $\sin \theta / \lambda$ dependence was nevertheless visible in the scatter plots of normalized $D_{\text {obs }}$. The result for TIBCAJ is shown in Fig. 4(e). Indeed, for a few of the compounds in the lower part of Table 1 the normalized $\left|D_{\text {obs }}\right|$ increased greatly with $\sin \theta / \lambda$.

Table 1 shows both Friedif stat $_{\text {and Friedif }}$ obs as obtained by the procedure described above for the compounds in FB2008. The calculation of these values requires knowledge of the chemical composition and wavelength together with an intensity data set containing the Friedel opposites. No structural model of the crystal is required. In all but one case (METWIS) the value of Friedif $f_{\text {obs }}$ is larger than Friedif ${ }_{\text {stat }}$. This is to be expected owing to the statistical and systematic uncertainties in the intensity data. The agreement between the two Friedif values decreases as one passes from the satisfactory data sets at the top of Table 1 to those less satisfactory ones at the bottom of Table 1. Clearly the value of Friedif ${ }_{\mathrm{obs}}$ is much affected by the statistical and systematic uncertainties in the intensity data. The value of Friedif stat $_{\text {assumes that the }}$ crystal structure is non-centrosymmetric, does not contain a centrosymmetric substructure, and the crystal is not twinned by inversion. For a crystal with a centrosymmetric structure or a crystal with a non-centrosymmetric structure twinned by inversion in a proportion of 50:50, the value of Friedif stat $_{\text {is }}$ zero. As a consequence, one may state with some confidence that:

(a) if Friedif obs $_{\text {is much lower than Friedif }}$ stat then the crystal structure is either centrosymmetric, or non-centrosymmetric with the crystal twinned by inversion in a proportion close to 50:50;

(b) if Friedif obs $_{\text {is close in value to Friedif }}$ stat as judged by the contents of Table 1, then the crystal is non-centrosymmetric and the intensity data set is satisfactory; and

(c) if Friedif obs $_{\text {is much larger than Friedif }}$ stat as judged by the contents of Table 1, then the data set is unsatisfactory or the chemical formula is erroneous.

Consequently analysis of an intensity data set by way of the values of Friedif stat $_{\text {and Friedif }}$ obs provides useful indications both of the space group and the quality of the intensity measurements.

Shmueli (2010) has suggested an interesting line of further development of the above procedure. As it stands, the 
procedure allows the detection of a centre of symmetry or of its absence to be established but does not attempt to identify amongst the noncentrosymmetric subgroups of a Laue class. However, using the tabulation of Shmueli \& Flack (2009), it is possible to identify classes of reflections which are centric in one subgroup but acentric in another. For example, it might well be possible to use the $h 0 l$ reflections in the monoclinic system to distinguish between the point groups 2 and $m$.

\section{Least-squares refinement}

Inclusion of the Flack (1983) parameter for the determination of absolute structure of noncentrosymmetric structures alongside the other crystallographic parameters in least-squares refinement has been routinely available for over 20 years (Watkin, 1991). Flack \& Bernardinelli (2000) provide a reasoned argument concerning confidence levels for the interpretation of the Flack (1983) parameter, and which can be used as a basis for secure publishable conclusions. Over recent years, a folklore has emerged which suggested that while Flack \& Bernardinelli (2000) provide rigorous criteria for the interpretation of the Flack (1983) parameter, the parameter is apparently indicative over a much larger range of values. This was supported by the study of 120 samples of known absolute configuration, containing no atoms heavier than oxygen, and measured with Mo $K \alpha$ radiation (Thompson \& Watkin, 2009).

\section{1. $A D$ refinement}

In $\S 2$ we showed that the ordinary $|\mathbf{F}|^{2}$ least-squares refinement of EZEQAB (and all the other compounds in the upper part of Table 1) gave quite satisfactory results. One is however entitled to wonder if, on changing the conditions of the least-squares refinement, whether a lower value of $R_{D}$ and a reduced scatter of points in the $D_{\text {obs }}$ versus $D_{\text {model }}$ plot could be obtained, leading to improved estimates of the atomic and crystal parameters. We have thus experimented along the following lines. The weighted sum of squares in a conventional refinement is given by

$$
S=\sum_{h k l}^{\text {all }} w(h k l)\left[\left|F(h k l)_{\mathrm{obs}}\right|^{2}-\left|F(h k l)_{\text {model }}\right|^{2}\right]^{2} .
$$

As the inverse of equations (1) and (2), $|\mathbf{F}(h k l)|^{2}$ and $|\mathbf{F}(\overline{h k l})|^{2}$ may be expressed in terms of $A(h k l)$ and $D(h k l)$ as

$$
\begin{aligned}
|F(h k l)|^{2} & =A(h k l)+\frac{1}{2} D(h k l), \\
|F(\overline{h k l})|^{2} & =A(h k l)-\frac{1}{2} D(h k l) .
\end{aligned}
$$

On collecting the data together into the classes of centric, unpaired acentric and paired acentric (Friedel pairs) reflections, using equations (8) and (9), and assuming $w(h k l)=$ $w(\overline{h k l})$ one readily obtains
Table 2

Residuals for structure refinements on WIGWUF, XICNED and SIHDET for five values $R_{A}=\sum_{h k l}^{\text {paired acentric }}\left|A_{\text {obs }}(h k l)-A_{\text {model }}(h k l)\right| / \sum_{h k l}^{\text {paired acentric }}\left|A_{\text {obs }}(h k l)\right|$, $R_{D}=\sum_{h k l}^{\text {paired acentric }}\left|D_{\text {obs }}(h k l)-D_{\text {model }}(h k l)\right| / \sum_{h k l}^{\text {paired acentric }}\left|D_{\text {obs }}(h k l)\right|$,

\begin{tabular}{llllll}
\hline REFCODE & $k$ & $R_{A}(\%)$ & $R_{D}(\%)$ & $w R_{A}^{2}(\%)$ & $w R_{D}^{2}(\%)$
\end{tabular}

$\begin{array}{lllll}0.01 & 5.0 & 48.0 & 6.2 & 42.5\end{array}$

$\begin{array}{lllll}1.0 & 5.0 & 48.0 & 6.2 & 42.2\end{array}$

$\begin{array}{lllll}1.0 & 5.3 & 48.0 & 6.2 & 40.9 \\ 10 & 5.3 & 47.6 & 6.7 & 38.8\end{array}$

$\begin{array}{lllll}00 & 7.1 & 47.1 & 10.3 & 38.8\end{array}$

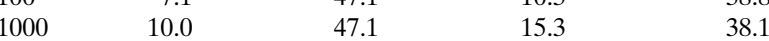

$\begin{array}{lllll}0.01 & 2.8 & 52.6 & 3.1 & 44.6\end{array}$

$\begin{array}{lllll}1.0 & 2.8 & 52.6 & 3.1 & 44.6\end{array}$

$\begin{array}{lllll}1.0 & 2.8 & 52.6 & 3.1 & 44.6 \\ 10 & 2.8 & 52.5 & 3.1 & 44.4\end{array}$

$\begin{array}{lllll}100 & 3.0 & 52.3 & 3.4 & 43.9\end{array}$

$\begin{array}{lllll}100 & 3.0 & 52.3 & 3.4 & 43.9 \\ 1000 & 3.1 & 52.2 & 3.7 & 43.7\end{array}$

$\begin{array}{lllll}0.01 & 7.5 & 96.5 & 8.0 & 96.4\end{array}$

$\begin{array}{lllll}0.01 & 7.5 & 96.5 & 8.0 & 96.4 \\ 1.0 & 7.5 & 96.5 & 8.0 & 96.4\end{array}$

$\begin{array}{lllll}10 & 7.5 & 96.5 & 8.0 & 96.4\end{array}$

$\begin{array}{lllll}100 & 7.6 & 96.4 & 8.1 & 96.3\end{array}$

$\begin{array}{lll}1000 & 12.0 & 95.3\end{array}$

$$
\begin{aligned}
S= & \sum_{h k l}^{\text {centric }} w(h k l)\left[\left|F(h k l)_{\mathrm{obs}}\right|^{2}-\left|F(h k l)_{\text {model }}\right|^{2}\right]^{2} \\
& +\sum_{h k l}^{\text {unpaired acentric }} w(h k l)\left[\left|F(h k l)_{\mathrm{obs}}\right|^{2}-\left|F(h k l)_{\text {model }}\right|^{2}\right]^{2} \\
& +\sum_{h k l}^{\text {paired acentric }} 2 w(h k l)\left[A(h k l)_{\mathrm{obs}}-A(h k l)_{\text {model }}\right]^{2} \\
& +k \sum_{h k l}^{\text {paired acentric }} \frac{1}{2} w(h k l)\left[D(h k l)_{\mathrm{obs}}-D(h k l)_{\text {model }}\right]^{2}
\end{aligned}
$$

For $k=1$ equation (10) is identical to equation (7) if the same weights $w(h k l)$ are used. However, on increasing the value of $k$ above 1, the weight of the Friedel-difference intensities becomes larger and their influence increases. It is not possible to undertake least-squares refinement to minimize the $S$ in equation (10) with conventional crystallographic software. However, with SMTK, the Small Molecule Tool Kit (Sadki \& Watkin, 2011), it is possible to minimize the $S$ of equation (10) for different values of $k$. In this way refinements were undertaken on the compounds WIGWUF, XICNED and SIHDET for a large range of values of $k$. In fact, the data for the refinements included only the paired acentric reflections whilst the centric and unpaired acentric reflections were omitted. The results are given in Table 2. All three structures show the same behaviour. A negligible contribution of the $D$ 's (i.e. $k=0.01$ ) to the sum of squares $S$ produces the same residuals as an 'ordinary' refinement (i.e. $k=1.0$ ). A large value of $k$ (i.e. $k=1000$ ) has the effect of diminishing $R_{D}$ and $w R_{D}^{2}$ very slightly whilst increasing $R_{A}$ and $w R_{A}^{2}$ to a greater extent. For WIGWUF and XICNED, the Flack parameter and its standard uncertainty were independent of the value of $k$. For SIHDET, the Flack parameter and its standard uncertainty changed by 0.03 on changing $k$. The $D_{\text {obs }}$ versus $D_{\text {model }}$ plots of these three compounds changed only very slightly or not at all between $k=0.01$ and $k=1000$. Moreover, the molecular geometry was very little altered between the low- 
and high- $k$ refinements. There seemed only to be some slight modification of the anisotropic atomic displacement parameters.

An independent check of the above results was made by using a slight variant of Parsons' restraints (Parsons, 2002; Parsons \& Flack, 2004) in the standard least-squares software CRYSTALS (Betteridge et al., 2003). In this procedure the complete set (centric, unpaired acentric and paired acentric) of intensity data is refined by least squares in the usual way with the inclusion of additional restraints (observations) in the weighted sum of squares. The additional restraints are none other than selected contributions to the final term of equation (10), the weighted sum of the squared (observed - model) differences of Friedel differences. The refinements were carried out on XICNED, DOPKID (Harvey et al., 2009) and MOKXEQ02 (Thompson et al., 2008) using a range of weights for the Friedel-difference intensities. As with the tests with $S M T K$ described above, the value of $R_{D}$ was unaffected by increasing the weights of the Friedel-difference intensities by a factor of $10^{4}$.

We conclude from these refinements that the fit to the observed intensities, and consequently the parameters of the atomic and crystal model, cannot be improved significantly by increasing the weight of the Friedel-difference intensities.

\section{2. $|F|^{2}$ refinement, scaling factor and weighting scheme}

It is tempting, but incorrect, to think that the problem revealed in the $D_{\text {obs }}$ versus $D_{\text {model }}$ plots of the compounds in the middle and lower parts of Table 1 might be resolved by applying an additional scale factor to the $D_{\text {model }}$ values. In fact, in the model of a non-centrosymmetric crystal structure twinned by inversion, such a scale factor is already available to the least-squares refinement by way of the Flack (1983) parameter. It is easy to show that $D_{\text {modelT }}(h k l)=$ $(1-2 x) D_{\text {modelU }}(h k l)$, where modelT and modelU refer to a crystal twinned or not by inversion, respectively, and $x$ is the Flack (1983) parameter. Consequently, a negative value of the Flack parameter corresponds to an increase, proportional to $(1-2 x)$, in the scale of the $D_{\text {modelu }}$ values. None of the compounds in Table 1 displayed a negative value of the Flack parameter of large absolute value corresponding to such an increased scale factor on the $D_{\text {model }}$ values.

In $\$ 2$, we observed that the data of compound SEZPUJ (and all those in the lower part of Table 1) have produced a satisfactory set of $A_{\mathrm{obs}}$ values whilst at the same time resulting in $D_{\text {obs }}$ values that are apparently valueless and lack information on the intensity differences between Friedel opposites due to resonant scattering. To say the least, it is both worrying and intriguing that the refined value of the Flack parameter 0.12 (11) for SEZPUJ does not reflect the disagreement between $D_{\text {obs }}$ and $D_{\text {model }}$ which is so manifest in Fig. 2. As a consequence we performed least-squares refinements on all of the compounds in the lower part of Table 1, starting each compound at a variety of values of the Flack (1983) parameter. In all cases, the value of the Flack parameter refined slowly but surely to a value close to the published value.
Changing the weights did not change this behaviour. Manifestly, the latter corresponds to a converged refinement and the published values of the Flack parameter are not arbitrary. It is also of relevance to examine the value of $R_{D}$ as a function of the value of the Flack parameter, keeping all other parameters fixed at their converged values. This is shown in Fig. 5 for five compounds in Table 1. One observes that for WIGWUF and YIDJIF, which occur in the upper part of Table 1 , there is a pronounced minimum of $R_{D}$ at the converged value of the Flack parameter. However, for SEZPUJ and YIFZAP, which occur in the lower part of Table 1, there is only an extremely shallow minimum in the value of $R_{D}$. The inescapable conclusion for these two compounds is that the published standard uncertainties of the Flack parameter are very grossly underestimated. CIKCUV, which occurs in the middle part of Table 1, shows an intermediate behaviour.

The value of the standard uncertainty of a least-squares parameter is calculated from the diagonal term of the inverse normal-equations matrix. The normal-equations matrix is composed entirely of terms which are sums of products (or squares for the diagonal terms) of derivatives of the squared structure-factor amplitudes with respect to the model of the crystal structure. In short, the terms of the inverse normalequations matrix are dependent solely on the crystal model and as such do not reflect the fit of the model to the observations. It is a common practice, which was certainly applied in the structure analyses of the compounds in Table 1, to then scale the standard uncertainties by the value of the weighted sum of squares as expressed in equations (7) and (10). This procedure seems to be unsatisfactory for the Flack (1983) parameter, as it is the only one to be conditioned exclusively by the final term in equation (10), the weighted sum of squared differences of the Friedel differences. A more appropriate calculation needs to be developed.

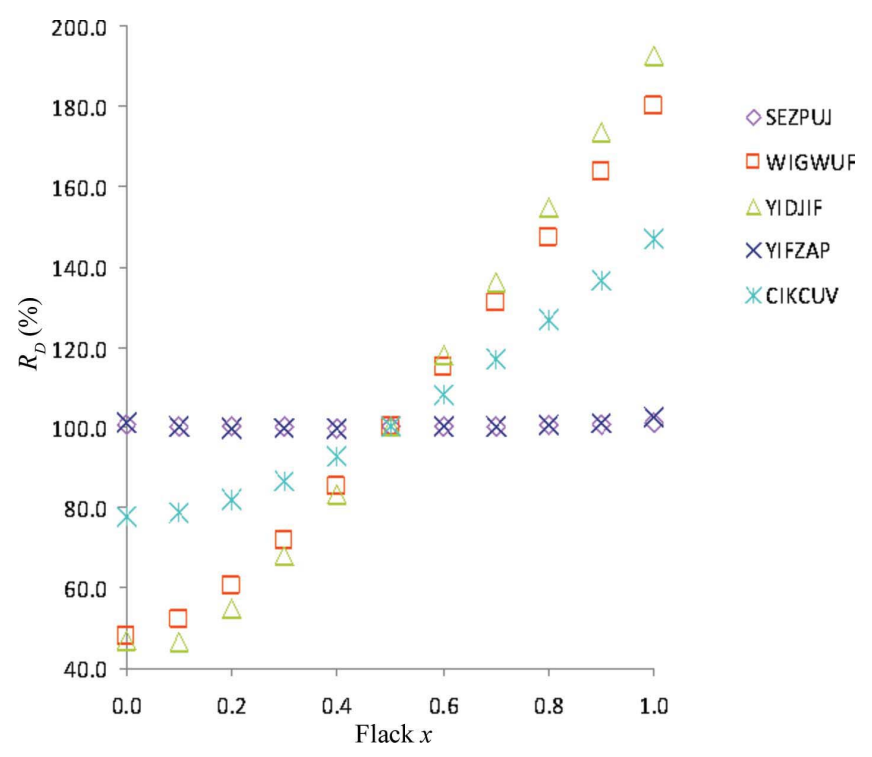

Figure 5

Plots of $R_{D}(\%)$ versus Flack $x$ for the compounds SEZPUJ, WIGWUF, YIDJIF, YIFZAP and CIKCUV. 


\section{Electron-density calculation}

The Fourier transform of the electron density of a crystal is the set of structure factors with amplitudes $|\mathbf{F}(h k l)|$ and phases $\varphi(h k l)$. As the electron density is a real function, these structure factors have the property that $|\mathbf{F}(h k l)|=|\mathbf{F}(\overline{h k l})|$ and $\varphi(h k l)=-\varphi(\overline{h k l})$. The intensity of the Bragg reflection $h k l$ is proportional to the square of the structure-factor amplitude. However, the particular structure factor concerned in the intensity is not the one derived only from the electron density but must also take account of the effects of resonant scattering. It follows that to calculate an electron density by Fourier transformation from experimental intensities, two steps have to be taken. The first is to correct the observed intensities for the effects of resonant scattering, and secondly to assign a phase to the observed structure-factor amplitude. Both of these calculations are undertaken by referring to an atomic model of the crystal structure. Larson (1976) gives details of one procedure for calculating the correction to the structure-factor amplitude $|\mathbf{F}(h k l)|$ of a single crystal, and Bernardinelli \& Flack (1985) extended this calculation to the case of a crystal twinned by inversion.

In the following we derive the appropriate Fourier coefficients for the (observed - model) difference electron density when using $A(h k l)$ and $D(h k l)$. We shall see that, in this form, the calculation of the electron-density Fourier coefficients is particularly simple. In the following, a subscript 'modelR' refers to a model value incorporating the effects of resonant scattering (both real and imaginary) and a subscript 'modelN' refers to a model value for which the resonant scattering contributions have been set to zero. The best estimate available for the contribution of the resonant scattering to $A_{\mathrm{obs}}$ is ( $\left.A_{\text {modelR }}-A_{\text {modelN }}\right)$. Consequently the amplitude of the Fourier coefficient for reflection $h k l$ to the (observed model) difference electron density is

$$
\begin{aligned}
\left|F_{\Delta \rho}(h k l)\right|= & \left\{A_{\mathrm{obs}}(h k l)-\left[A_{\text {modelR }}(h k l)-A_{\text {modelN }}(h k l)\right]\right\}^{1 / 2} \\
& -\left[A_{\text {modelN }}(h k l)\right]^{1 / 2} .
\end{aligned}
$$

It is important to note that the amplitude in equation (11) is independent of both $D_{\text {obs }}$ and $D_{\text {model }}$. The appropriate phase angle for this Fourier coefficient is $\varphi_{\text {modelN }}(h k l)$. On taking first-order Taylor expansions for the various terms in equation (11) and simplifying, one finds

$$
\left|F_{\Delta \rho}(h k l)\right| \simeq\left[A_{\mathrm{obs}}(h k l)\right]^{1 / 2}-\left[A_{\text {modelR }}(h k l)\right]^{1 / 2}
$$

valid for

$$
\begin{gathered}
\left|\left(A_{\text {modelR }}-A_{\text {modelN }}\right) / A_{\text {obs }}\right| \ll 1, \\
\left|\left(A_{\text {modelR }}-A_{\text {modelN }}\right) / A_{\text {modelR }}\right| \ll 1 .
\end{gathered}
$$

\section{The $A$ - and $D$-Patterson functions}

Our continuing work (Shmueli \& Flack, 2010) on intensity statistics leads us directly to the novel use of the Patterson function presented in this section. The Fourier transform of $A$ is known in crystallography as the Patterson function. A review of Patterson functions relevant to the current article is that of Rossmann \& Arnold (2001), as this deals explicitly with the Pattersons derived from $A$ and $D$. As shown in Shmueli \& Flack (2010), based on results of Okaya \& Pepinksy (1955) and Rossmann \& Arnold (2001), one may write $A$ and $D$ in terms of interatomic vectors in the following way,

$$
\begin{aligned}
A(h k l)= & \sum_{i=1}^{N} \sum_{j=1}^{N}\left[\left(f_{i}^{\mathrm{o}}+f_{i}^{\prime}\right)\left(f_{j}^{\mathrm{o}}+f_{j}^{\prime}\right)+f_{i}^{\prime \prime} f_{j}^{\prime \prime}\right] \cos 2 \pi \\
& \times\left[h\left(x_{i}-x_{j}\right)+k\left(y_{i}-y_{j}\right)+l\left(z_{i}-z_{j}\right)\right], \\
D(h k l)= & 2 \sum_{i=1}^{N} \sum_{j=1}^{N}\left[\left(f_{i}^{\mathrm{o}}+f_{i}^{\prime}\right) f_{j}^{\prime \prime}-f_{i}^{\prime \prime}\left(f_{j}^{\mathrm{o}}+f_{j}^{\prime}\right)\right] \sin 2 \pi \\
& \times\left[h\left(x_{i}-x_{j}\right)+k\left(y_{i}-y_{j}\right)+l\left(z_{i}-z_{j}\right)\right] .
\end{aligned}
$$

The form of the $A$-Patterson for space groups $P 1$ and $P \overline{1}$ is

$$
P_{A}(u v w)=\frac{2}{V} \sum_{h k l}^{\text {hemisphere }} A(h k l) \cos 2 \pi(h u+k v+l w) .
$$

As can be seen in equation (12), the $A$-Patterson comprises peaks situated at the extremities of the interatomic vectors $\left(x_{i}-x_{j}, y_{i}-y_{j}, z_{i}-z_{j}\right)$ with peak heights proportional to $\left[\left(f_{i}^{\mathrm{o}}+f_{i}{ }^{\prime}\right)\left(f_{j}^{\mathrm{o}}+f_{j}{ }^{\prime}\right)+f_{i}^{\prime \prime} f_{j}^{\prime \prime}\right]$. The $A$-Patterson is centrosymmetric as the peak height of the vector between atoms $j$ and $i$ is identical to that between atoms $i$ and $j$. In most older treatments of the Patterson function both the real and the imaginary resonant scattering contributions to $A$ were ignored and an approximate Patterson was calculated directly using either the individual $|\mathbf{F}(h k l)|^{2}$ or $|\mathbf{F}(\overline{h k l})|^{2}$ of the observed intensities. The use of the Patterson function for crystal structure solution has now been largely superseded by other methods.

The Fourier transform of $D$ has never been used in structure solution but a simulation by Woolfson (see Rossmann \& Arnold, 2001) confirms that it has interesting properties. The form of the $D$-Patterson for space group $P 1$ is

$$
P_{D}(u v w)=\frac{2}{V} \sum_{h k l}^{\text {hemisphere }} D(h k l) \sin 2 \pi(h u+k v+l w) .
$$

As can be seen from equation (13), the $D$-Patterson again has peaks at the extremities of vectors between atoms $i$ and $j$ with heights proportional to $\left[\left(f_{i}^{\mathrm{o}}+f_{i}^{\prime}\right) f_{j}^{\prime \prime}-\left(f_{j}^{\mathrm{o}}+f_{j}^{\prime}\right) f_{i}^{\prime \prime}\right]$. This means that interatomic vectors between atoms of the same chemical element correspond to peaks of zero height in the $D$-Patterson. Moreover the $D$-Patterson is antisymmetric, as the peak height of the vector between atoms $i$ and $j$ is the negative value of that between atoms $j$ and $i$. For a molecule with a single resonant atom and a host of equal non-resonant atoms, the $D$-Patterson shows an antisymmetric image of the molecule. The above analysis further shows that there are two causes making the difference between the intensities of Friedel opposites small. The first is that $f^{\prime \prime}$ is small compared to $f^{\circ}$. The second is that interatomic vectors between different atoms of the same chemical element do not contribute to the difference intensity. We further point out that the $D$-Patterson 
provides a means of structure validation that is not available by way of an electron-density calculation, for, as seen in $\$ 5$, the latter is independent of the Friedel-difference intensities.

An $\left(A_{\text {obs }}-A_{\text {model }}\right)$-Patterson evaluated at the end of structure refinement will reveal missing or superfluous interatomic vectors in the final structure model. That this Patterson is preferable to a difference electron-density calculation may be understood by the bias from the model introduced into the latter. Firstly, for the difference electrondensity calculation, the phase of the observed structure factor is arbitrarily set to that of the calculated structure factor whereas for the calculation of the Patterson, phases are not required. Secondly, for twinned crystals, for a difference electron-density calculation, the observed intensities need to be detwinned using the twin fractions of the model whereas an ( $\left.A_{\text {obs }}-A_{\text {model }}\right)$-Patterson does not require any detwinning. Thirdly, for the difference electron-density calculation, the $A_{\text {obs }}$ values need to be corrected for the resonant scattering contribution as detailed in $§ 5$. This again introduces a bias in the corrected observed quantities. Fourthly, random uncertainties may engender $A_{\text {obs }}$ values that are negative, leading to problems in taking the square roots in equation (11). In the same vein, to avoid bias, it may well be of interest to calculate this ( $\left.A_{\text {obs }}-A_{\text {model }}\right)$-Patterson for intensity data averaged only in the space group $P 1$ and retaining all space-group-absent reflections. In this way all assumptions as to the crystal's symmetry are confined to the model.

As examples, the $A_{\text {obs }^{-}}, A_{\text {model }^{-}}$and $\left(A_{\text {obs }}-A_{\text {model }}\right)$ Pattersons have been calculated with $S M T K$ (Sadki \& Watkin, 2011) for the compounds XICNED and DOPKID (Harvey et $a l$., 2009) using only the measurements of $A(h k l)$. For XICNED, at least the first 20 highest peaks of the $A_{\text {obs }^{-}}$and

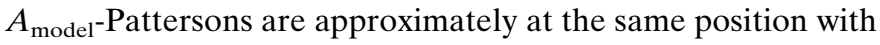

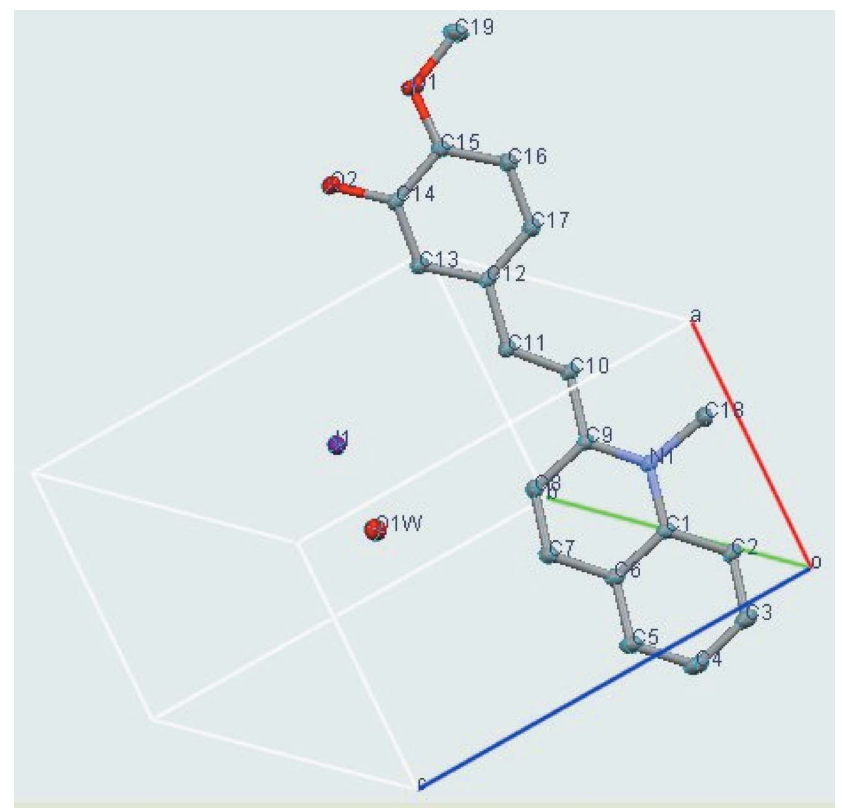

Figure 6

Molecular structure of XICNED in its unit cell. the same intensity (peak Q1 at the origin has heights of 5267 and 5241; peak Q2 has heights of 480 and 478; and peak Q18 has heights of 328 and 330 , respectively). In the ( $A_{\text {obs }}-$ $A_{\text {model }}$ )-Patterson the first three highest peaks (heights 46.2 , $45.9,34.6)$ do not occur at the positions of interatomic vectors in the structure. Peak 4 (height 29.9) occurs at the origin. The lowest trough has a value of -19.1 and again is not at the position of an interatomic vector. DOPKID is similar. The first 31 highest peaks of the $A_{\mathrm{obs}^{-}}$and $A_{\text {model-Pattersons }}$ are approximately at the same position with the same intensity (peak Q1 at the origin has heights of 2336 and 2316; peak Q2 has heights of 577 and 562; and peak Q28 has heights of 151 and 145; the lowest troughs have values of -706 and -708 , respectively). In the $\left(A_{\text {obs }}-A_{\text {model }}\right)$-Patterson the first highest peak (height 20.1) is at the origin. The lowest trough (height -62.7) does not occur at the position of an interatomic vector in the structure. Such values indicate that the structure analyses of XICNED and DOPKID are satisfactory. CRYSTALS (Betteridge et al., 2003) was used to check these results on DOPKID and gave essentially the same results as $S M T K$, despite the fact that the former uses all reflections (centric, unpaired acentric and averaged paired acentric) whereas $S M T K$ uses only paired acentric reflections.

Various $D_{\text {obs }}$ - and $\left(D_{\text {obs }}-D_{\text {model }}\right)$-Pattersons have been calculated for XICNED and EDUZOT. The molecular structure of XICNED is shown in Fig. 6 and the intensity data are of good quality as the compound appears in the upper part of Table 1. The molecule is planar and there is an atom of iodine (a strong resonant scatterer) in the structure not in the plane of the molecule. Fig. 7 shows a contour plot of a selected plane of the positive peaks in the $D_{\text {obs }}$-Patterson of XICNED. The atoms of the molecule are clearly visible imaged by the resonant iodine atom. Fig. 7 is thus a demonstration with real data that the $D_{\mathrm{obs}}$-Patterson is capable of showing a complete image of the molecule as in Woolfson's (see Rossmann \& Arnold, 2001) simulation. Fig. 8 shows in projection both the peaks (red) and the troughs (light green) of the $D_{\text {obs }}$-Patterson of XICNED, clearly demonstrating its antisymmetric nature. Also shown in Fig. 8 are the peaks (blue) and the troughs (brown) of the $\left(D_{\text {obs }}-D_{\text {model }}\right)$-Patterson of XICNED. These are not in positions overlapping any interatomic vector. As a comparison we show one plot for EDUZOT. The molecule is roughly planar and the intensity data are of much lower quality as the compound appears in the lower part of Table 1. For EDUZOT the $D_{\text {obs }}$ versus $D_{\text {model }}$ plot shows the data to be spread along the $D_{\text {obs }}$ axis with little or no relation between the $D_{\text {obs }}$ and $D_{\text {model }}$ values. Fig. 9 shows a $D_{\text {obs }}$-Patterson of EDUZOT in the plane of the molecule. There is no recognizable feature in this Patterson nor in that of the $\left(D_{\text {obs }}-\right.$ $D_{\text {model }}$ )-Patterson, not illustrated.

We now also present some Patterson maps for cases where the model has been made erroneous in some way or another. It is, however, beyond the scope of the present article to present an in-depth interpretation and study of the positions and magnitudes of the peaks and troughs in these various Patterson functions. Fig. 10 shows a composite threedimensional view of various Patterson functions for XICNED 
for which the C9 atom of the model has been displaced by $0.63 \AA$ along a from its refined position. The contour lines are the peaks of the molecular plane of the $D_{\text {obs }}$-Patterson, with the peaks of the $\left(D_{\text {obs }}-D_{\text {model }}\right)$-Patterson in blue and those of the $\left(A_{\mathrm{obs}}-A_{\text {model }}\right)$-Patterson in mauve. Fig. 11 is a similar composite three-dimensional view of various Patterson functions for XICNED for a model where the C9 atom has been removed. The contour lines are the peaks of the molecular plane of the $D_{\mathrm{obs}}$-Patterson, with the in-plane peaks of the ( $\left.D_{\text {obs }}-D_{\text {model }}\right)$-Patterson as grey spheres and those of the ( $\left.A_{\text {obs }}-A_{\text {model }}\right)$-Patterson as red spheres. The C9 atom does not appear in the $\left(D_{\text {obs }}-D_{\text {model }}\right)$-Patterson. In a further false solution of XICNED, the model has been modified so that atom $\mathrm{N} 1$ has been made into a carbon atom. Fig. 12 shows the composite three-dimensional view. The contour lines are the peaks of the molecular plane of the $D_{\text {obs }}$-Patterson, with

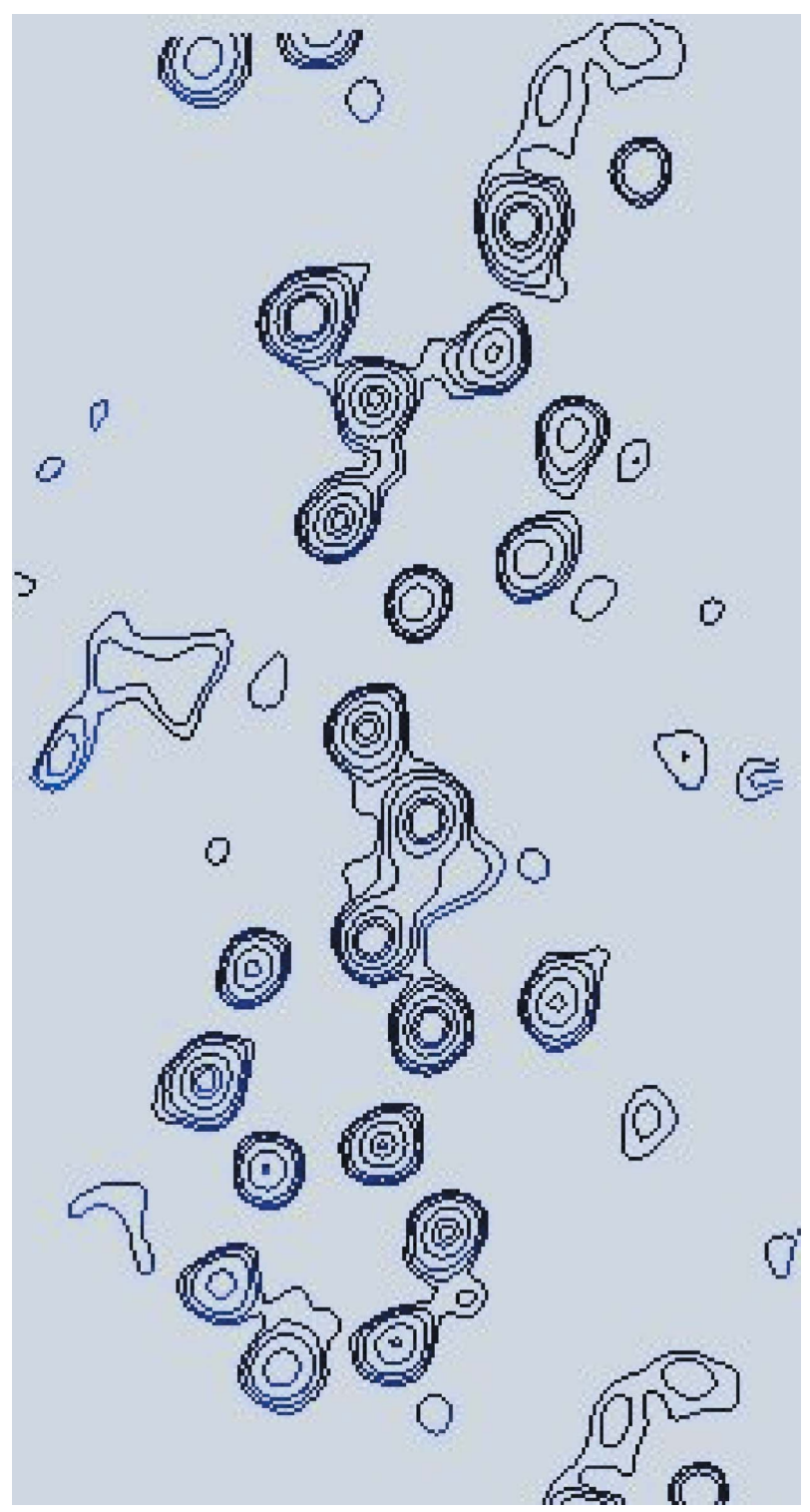

Figure 7

Contour map of a selected plane of the $D_{\text {obs }}$-Patterson of XICNED showing the molecular structure. Only positive peaks are drawn. the in-plane peaks of the $\left(A_{\mathrm{obs}}-A_{\text {model }}\right)$-Patterson as mauve spheres.

As a final example of an erroneous structure solution, we present a case of a partial-polar ambiguity (Kuchta \& Parkin, 1998). The essence of this pitfall is that a proposed structure model is essentially correct but a part of the model is inverted in a point with respect to the true structure. The intensity data for YINLEM are excellent, like those of EZEQAB. The final $R_{D}$ value is $43.5 \%$ and the data points lie around a line of slope 1.0 with zero intercept in the $D_{\text {obs }}$ versus $D_{\text {model }}$ plot. However, an initial structure solution of YINLEM (Larsonneur et al., 1994) of low $R$ value showed an image of the $\mathrm{Cl}$ atom on the opposite side of the $\mathrm{Au}$ atom to its true position, i.e. at position $0.25,0.25,0.21342$ instead of $0.25,0.25,0.78658$. All other

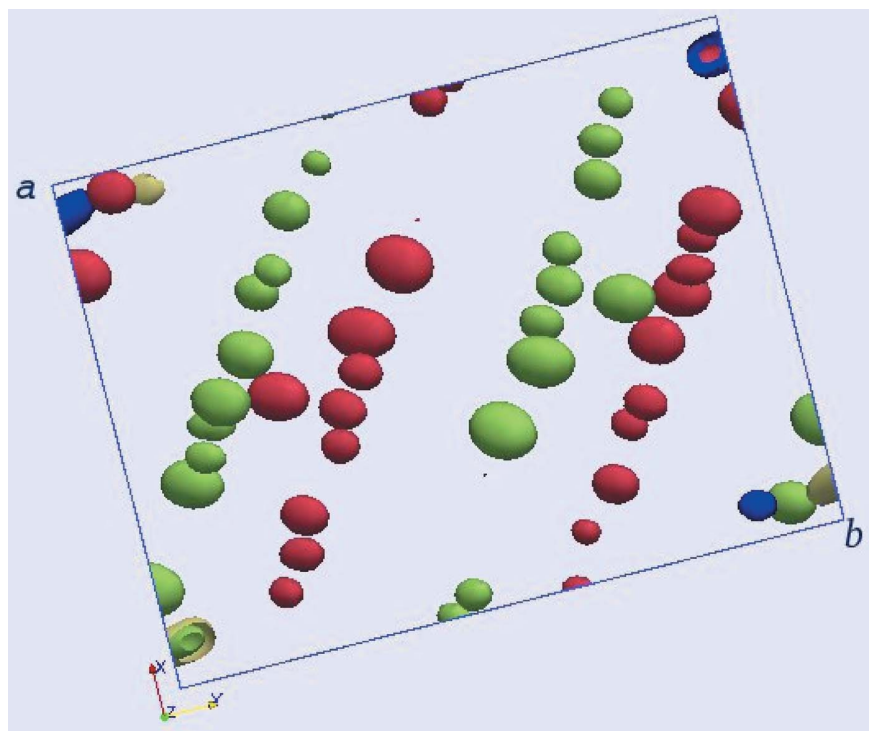

Figure 8

A projection of the $D_{\mathrm{obs}}$-Patterson of XICNED showing both positive (red) and negative (green) peaks demonstrating the antisymmetric nature of this function. Also shown are the positive (blue) and negative (brown) peaks of the $\left(D_{\text {obs }}-D_{\text {model }}\right)$-Patterson of XICNED.

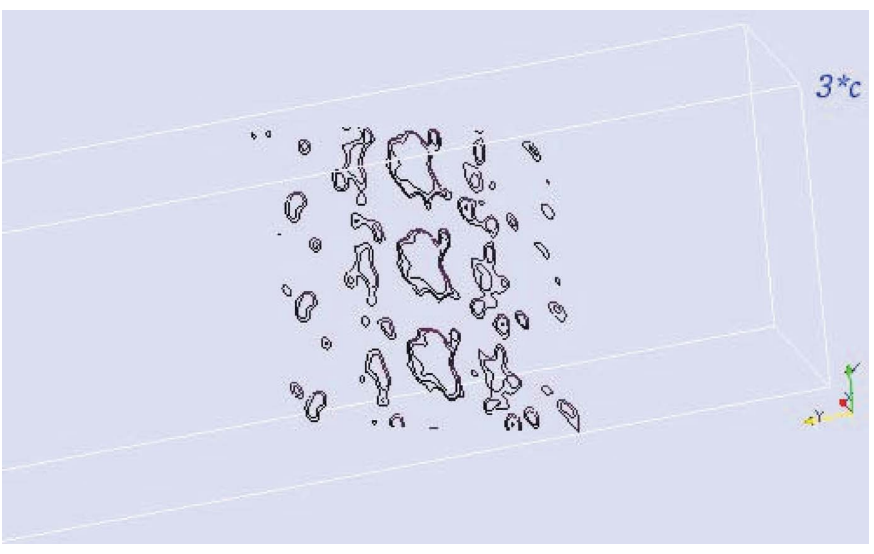

Figure 9

Contour plot in the plane of the molecule of the $D_{\text {obs }}$-Patterson of EDUZOT. There are no recognizable features of the molecular interatomic vectors in this map. 


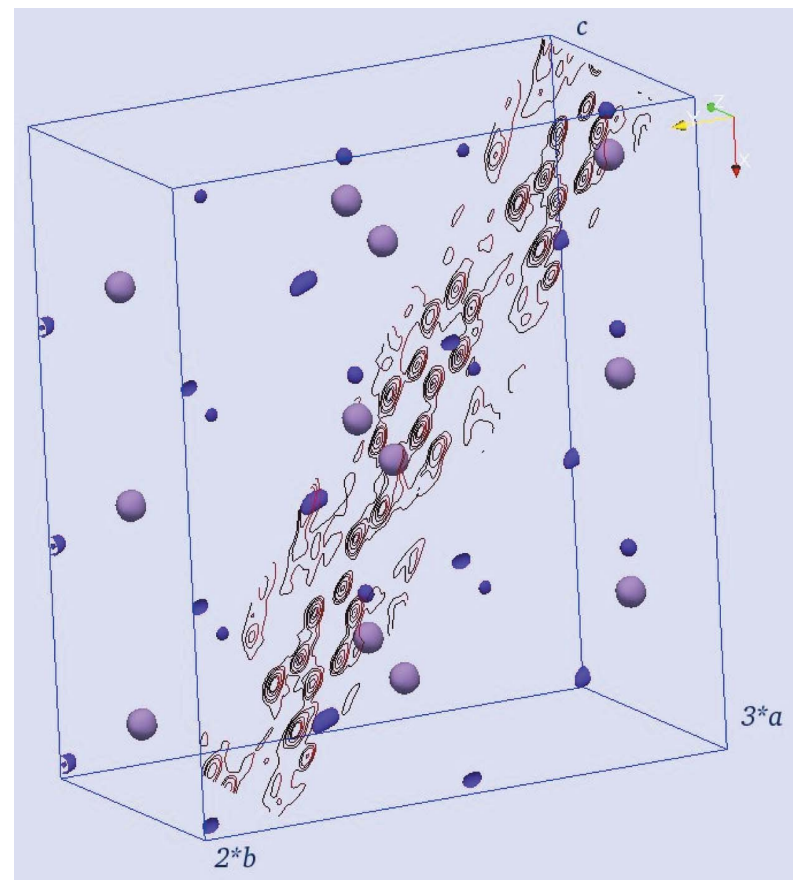

Figure 10

A composite three-dimensional view of various Patterson functions for XICNED for which the $\mathrm{C} 9$ atom of the model has been displaced by $0.63 \AA$ along a from its refined position. The contour lines are the peaks of the molecular plane of the $D_{\text {obs }}$-Patterson, with the peaks of the $\left(D_{\text {obs }}\right.$ $\left.-D_{\text {model }}\right)$-Patterson in blue and those of the $\left(A_{\text {obs }}-A_{\text {model }}\right)$-Patterson in mauve.

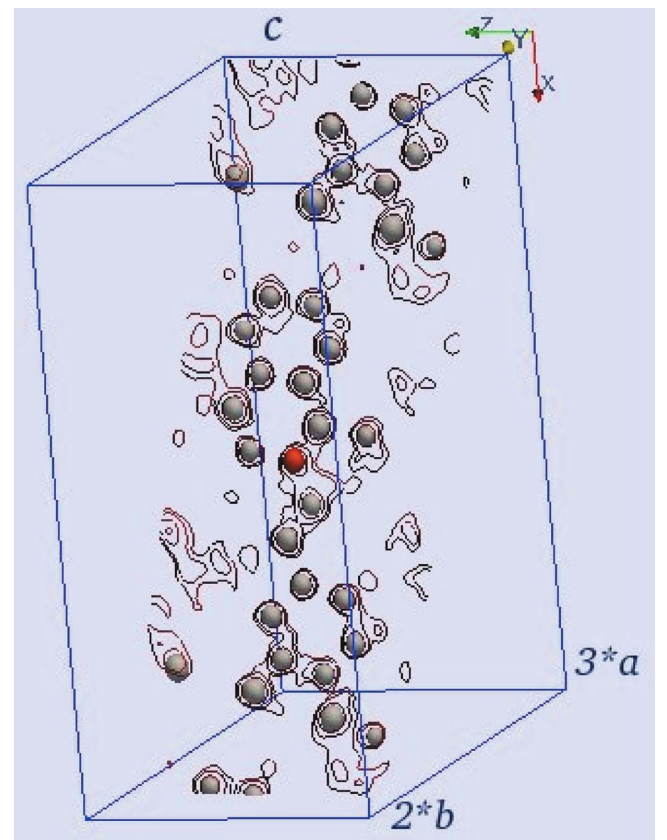

Figure 11

Patterson functions of XICNED in which the $\mathrm{C} 9$ atom has been removed from the model. The contour lines are the peaks of the molecular plane of

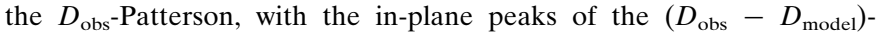
Patterson as grey spheres and those of the $\left(A_{\text {obs }}-A_{\text {model }}\right)$-Patterson as red spheres. The C9 atom does not appear in the $\left(D_{\text {obs }}-D_{\text {model }}\right)$ Patterson. atoms were correctly located. It is interesting to note in passing that the $D_{\text {obs }}$ versus $D_{\text {model }}$ plot for the model with the $\mathrm{Cl}$ atom in the wrong position shows more scatter than the one with the $\mathrm{Cl}$ atom in its proper place. Fig. 13 shows a composite three-dimensional view of various Patterson functions of YINLEM for a model in which the chlorine atom is in the erroneous position. The peaks (blue) and troughs (green) of the $\left(A_{\text {obs }}-A_{\text {model }}\right)$-Patterson are shown in Fig. 13 with the peaks (red) and troughs (light red) of the ( $\left.D_{\text {obs }}-D_{\text {model }}\right)$ Patterson. Such plots reveal the partial-polar ambiguity.

\section{The counting of reflections}

For the purposes of validating crystal structure determinations it would be of the greatest help if, for reflections obtained by merging in the crystal class, that, apart from the total number of unique reflections, the counts of the following reflections could be reported: (a) centric reflections; $(b)$ pairs of acentric reflections where both $h k l$ and $\overline{h k l}$ have been measured; and (c) acentric reflections where only one of $h k l$ and $\overline{h k l}$ has been measured.

It is of particular interest that reflections in classes $(a)$ and (c) should not be counted together. One measure of the quality of the data is the number of reflections in class $(c)$. This should be as low as possible. Shmueli \& Flack (2009) provide a useful table for the classification of reflections into centric and acentric classes, as well as general and special classes.

For example, for compound CIJWUO (see FB2008), space group $o P 22_{1} 2_{1} 2_{1}$, the paper and the CIF state 'Flack (1983), 780 Friedel pairs', whilst the Checkcif/PLATON report (http:// checkcifiucr.org/; Spek, 2003) contains a G-level alert indicating an estimated 391 Friedel pairs for 3120 symmetry unique reflections and a total number of reflections of 3511 . Of

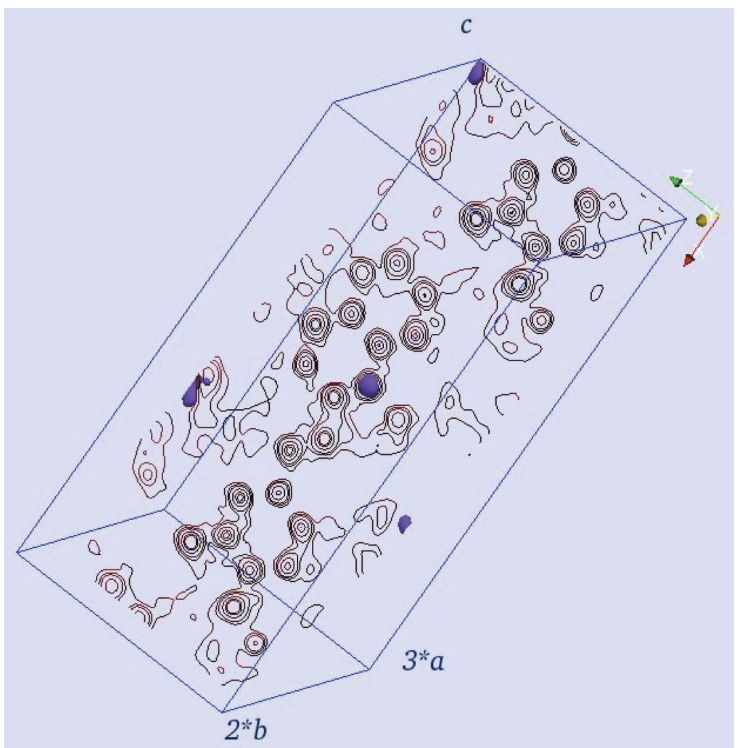

Figure 12

Patterson functions of XICNED in which the model has been modified so that atom $\mathrm{N} 1$ has been made into a carbon atom. The contour lines are the peaks of the molecular plane of the $D_{\text {obs }}$-Patterson, with the in-plane peaks of the ( $\left.A_{\mathrm{obs}}-A_{\text {model }}\right)$-Patterson as mauve spheres. 
the 3511 symmetry-independent reflections in the CIF file containing the intensity data, there are 709 centric reflections, 414 pairs of Friedel opposites and 1974 acentric reflections for which the Friedel opposite was not measured. Whilst 414 Friedel pairs might seem adequate at first sight, one has to compare that value with 1974 acentric reflections for which the Friedel opposite was not measured.

\section{Concluding remarks}

The analysis of a set of data and a crystal structure solution as developed in the current work relies on the measurement of a complete set of diffraction intensities, especially one including all pairs of Friedel opposites. It has been shown how the scattergrams of $D_{\text {obs }}$ versus $D_{\text {model }}$ and the $\left(D_{\text {obs }}-D_{\text {model }}\right)$ Patterson provide useful information for the validation of the structure analysis. In $\S 6$ we briefly mentioned that it might prove of use to calculate the ( $\left.A_{\mathrm{obs}}-A_{\text {model }}\right)$-Patterson from intensity data that had been averaged for the space group $P 1$ rather than in the space group selected for the structure solution. Indeed the fact that the intensity file, published as a supplementary file for Acta Crystallographica B, C and E, contains data averaged and merged for the point group selected for the crystal structure solution restricted some of the analyses that we wished to undertake. Intensity data merged only for the space group $P 1$ would have been of far greater use and flexibility.

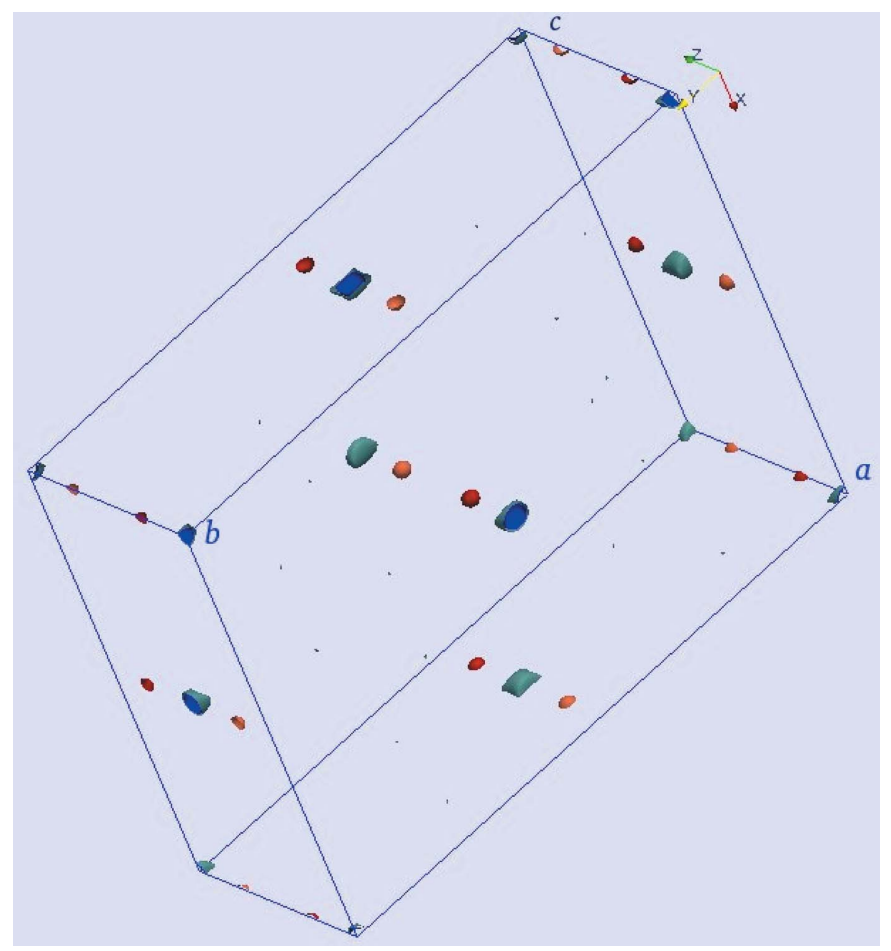

Figure 13

A composite three-dimensional view of various Patterson functions of YINLEM for a model in which the chlorine atom is in the erroneous position. The peaks (blue) and troughs (green) of the $\left(A_{\text {obs }}-A_{\text {model }}\right)$ Patterson are shown with the peaks (red) and troughs (light red) of the $\left(D_{\text {obs }}-D_{\text {model }}\right)$-Patterson.
It has also become apparent through our methods of validation that whereas all the data sets that we analysed provided a satisfactory set of average Friedel intensities $A(h k l)_{\text {obs }}$, only approximately one third of them also provided satisfactory values of $D(h k l)_{\text {obs }}$. Another one third of the data sets provided poor $D(h k l)_{\text {obs }}$ values which were dominated by random and systematic effects unrelated to resonant scattering. The final one third of the data sets was intermediate in their behaviour. The poor $D(h k l)_{\text {obs }}$ values are undoubtedly due to sample preparation, machine alignment, measurement protocols and data-reduction procedures. There is hence an urgent need that these be studied in detail and improved.

It often occurs in practice that authors of non-centrosymmetric crystal structures containing only light atoms are requested by editors or referees to average intensities of Friedel opposites. The basis of this request is the perception that the resonant scattering contribution from atoms lighter than $\mathrm{F}$ is so small as to be insignificant. With the greatest possible respect for this historical approach, we submit that it is outdated. An eminently more objective approach would be to require authors to submit evidence similar to that used in the current paper to evaluate the real contribution of the resonant scattering to the observed difference of intensity of the Friedel opposites.

One aspect of this analysis that we have as yet not completely resolved is to provide an understanding of why data sets which provide a poor fit of $D_{\text {obs }}$ to $D_{\text {model }}$ nevertheless provide acceptable values of the Flack (1983) parameter with its standard uncertainty and in the analysis of Hooft et al. (2008). The final paragraph of $\$ 4.2$ explains why the standard uncertainty is underestimated but does not provide a more objective method of estimation. The cause of the apparently acceptable values of the Flack parameter itself remains a mystery at the moment.

G. Bernardinelli, A. Linden, U. Shmueli and A. F. Williams are thanked for commenting on a draft version of this paper.

\section{References}

Allen, F. H. (2002). Acta Cryst. B58, 380-388.

Bernardinelli, G. \& Flack, H. D. (1985). Acta Cryst. A41, 500-511.

Betteridge, P. W., Carruthers, J. R., Cooper, R. I., Prout, K. \& Watkin, D. J. (2003). J. Appl. Cryst. 36, 1487.

Flack, H. D. (1983). Acta Cryst. A39, 876-881.

Flack, H. D. \& Bernardinelli, G. (2000). J. Appl. Cryst. 33, 1143-1148. Flack, H. D. \& Bernardinelli, G. (2008). Acta Cryst. A64, 484-493.

Flack, H. D. \& Shmueli, U. (2007). Acta Cryst. A63, 257-265.

Harrison, W. T. A., Simpson, J. \& Weil, M. (2010). Acta Cryst. E66, e1-e2.

Harvey, J. S., Malcolmson, S. J., Dunne, K. S., Meek, S. J., Thompson, A. L., Schrock, R. R., Hoveyda, A. H. \& Gouverneur, V. (2009). Angew. Chem. Int. Ed. 48, 762-766.

Hooft, R. W. W., Straver, L. H. \& Spek, A. L. (2008). J. Appl. Cryst. 41, 96-103.

IUCr Editorial Office (2010). Acta Cryst. E66, e21-e22.

Kuchta, M. C. \& Parkin, G. (1998). New J. Chem. 22, 523-530.

Larson, A. C. (1976). Crystallographic Computing Techniques, edited by F. R. Ahmed, co-editors K. Huml \& B. Sedlacek, pp 396-398. Copenhagen: Munksgaard. 
Larsonneur, A.-M., Turpin, R., Castan, P. \& Bernardinelli, G. (1994). Inorg. Chim Acta, 227, 85-90.

Looijenga-Vos, A. \& Buerger, M. J. (2002). International Tables for Crystallography, Vol. A, Space-Group Symmetry, edited by Th. Hahn, Part 3, pp. 43-54. Dordrecht: Kluwer Academic Publishers. Marsh, R. E. (2009). Acta Cryst. B65, 782-783.

Okaya, Y. \& Pepinksy, R. (1955). Computing Methods and the Phase Problem in X-ray Crystal Analysis, p. 276. Oxford: Pergamon Press.

Parsons, S. (2002). Contribution E0043/08.01.11, Precise Absolute Structure Determination for Light Atom Structures, to the American Crystallographic Association Meeting, San Antonio, Texas, 25-30 May 2002.

Parsons, S. \& Flack, H. (2004). Acta Cryst. A60, s61.

Rossmann, M. G. \& Arnold, E. (2001). International Tables for Crystallography, Vol. B, Reciprocal Space, edited by U. Shmueli, ch. 2.3, pp. 235-263. Dordrecht: Kluwer Academic Publishers.
Sadki, M. \& Watkin, D. J. (2011). J. Appl. Cryst. 44, doi:10.1107/ S0021889810042470.

Shmueli, U. (2010). Personal communication.

Shmueli, U. \& Flack, H. D. (2009). Acta Cryst. A65, 322-325.

Shmueli, U. \& Flack, H. D. (2010). Acta Cryst. A66, 669-675.

Shmueli, U., Schiltz, M. \& Flack, H. D. (2008). Acta Cryst. A64, 476483.

Spek, A. L. (2003). J. Appl. Cryst. 36, 7-13.

Thompson, A. L. \& Watkin, D. J. (2009). Tetrahedron Asymmetry, 20, 712-717.

Thompson, A. L., Watkin, D. J., Gal, Z. A., Jones, L., Hollinshead, J., Jenkinson, S. F., Fleet, G. W. J. \& Nash, R. J. (2008). Acta Cryst. C64, o649-o652.

Watkin, D. J. (1991). Crystallographic Computing 5, edited by D. Moras, A. D. Podjarny \& J. C. Thierry, pp. 93-103. Oxford University Press. 